\title{
On Separation of Phases in One-Dimensional Gases
}

\author{
Kurt Johansson \\ Department of Mathematics, Royal Institute of Technology, S-10044 Stockholm, Sweden. \\ e-mail: kurtj@math.kth.se
}

Received: 15 November 1993

\begin{abstract}
We prove that in a one-dimensional gas in the canonical ensemble with pair interaction $A / r^{\gamma}-B / r^{2}, \gamma>2$, we have a separation of phases at sufficiently low temperatures. The same combinatorial framework can be used for both lattice and continuous models. A rather precise bound on the critical temperature in a $1 / r^{2}$ lattice gas is obtained when the nearest neighbour coupling is large. The interface between the two phases is defined and investigated.
\end{abstract}

\section{Introduction}

There are many results on phase transitions in lattice models in all dimensions. For continuous models however there are very few results, see [Ru2] or [Is], appendix. It is still an open problem to prove that there is a phase transition in a 3-dimensional continuous gas with a Lennard-Jones interaction, $A / r^{12}-B / r^{6}$, see [Si], problem 7. In view of this it seems worthwhile to try to establish the existence of a phase transition in a 1-dimensional continuous gas in particular in the non-hard core case. A phase transition in one dimension requires a long-range interaction, which makes the argument complicated, but on the other hand the difference between a lattice and a continuous gas should be least in one dimension.

We need an energy-entropy argument which is not so sensitive to the exact location of the particles, i.e. if we move the particles slightly the argument should still be valid. In two previous papers such a method was developed and it was proved that in a lattice gas [Jo1] or in a hard-core continuous gas [Jo2], in the canonical ensemble, with attractive pair interaction $-1 / r^{\alpha}, 1<\alpha \leq 2$, there is condensation at sufficiently low temperatures. Here condensation means that for a large system, with high probability, the gas has non-uniform density, a separation of phases occurs. A heuristic argument is given in the introduction to [Jo1].

In this paper these results will be extended to a continuous gas without hardcore. The pair interaction will be of Lennard-Jones type, $A / r^{\gamma}-B / r^{2}, \gamma>2$. This model is more unlike a lattice gas and is more difficult to deal with than the hard-core continuous gas. 
In the papers [Jo1] and [Jo2] different combinatorial arguments were used for the lattice and the continuous case. In the present paper the same combinatorial framework (entropy estimate) is used for both the lattice and the continuous gas, only the energy estimates are different. Also the rearrangement (condensation) procedure is simpler than those used in [Jo1] and [ $\mathrm{J}_{\mathrm{O} 2}$ ]. Furthermore the phase separation and the interface are more carefully defined.

For the lattice gas corresponding to an $1 / r^{2}$-Ising model, a rather precise estimate of the critical temperature is obtained in the limit when the nearest neighbour coupling becomes large. This has been investigated also in [ACCN] and [IN]. To be able to deal with continuous models one has to leave the strictly ferromagnetic models, so the results for the lattice gas (Ising model) are valid even if not all couplings $J_{r}$ are $\geq 0$, provided $J_{r} \sim 1 / r^{2}$ as $r \rightarrow \infty$.

The organization of the paper is as follows. In Sect. 1 we define the models considered, write the continuous gas as a lattice model, define what we mean by a phase separation and state our results. Section 2 contains the energy-entropy argument for what we call a general lattice gas. In Sects. 3 and 4 the energy estimates for the lattice and continuous gas respectively are proved. Together with the general energyentropy argument of Sect. 2, they establish the results in Sect. 1. The last section contains the proof of an energy estimate used for the continuous gas in Sect. 4. To prove this estimate we must show that the probability that we have very many particles in a short interval, or in a sequence of short intervals is small.

\section{Definitions and Results}

\subsection{The General Lattice Gas}

A continuous gas and a lattice gas on a line can both be written as special cases of what we call a general lattice gas, which is defined as follows. Consider a one-component one-dimensional lattice gas where we allow multiple occupation at a lattice point. We will work in the canonical ensemble so we have a fixed number $N$ of particles in our box $\Lambda=\mathbb{Z} \cap[0, L)$. Let $L=[N / \varrho]$, where $\varrho \in \mathbb{R}^{+}$is given, $\varrho$ is the overall density. For our purposes the following boundary conditions are convenient: all lattice points in $\mathbb{Z} \cap(-\infty, 0)$ are occupied by a single particle, and all lattice points in $\mathbb{Z} \cap[L, \infty)$ are empty. This means that our basic configuration space is

$$
Q_{N, \varrho}=\left\{\underline{n} \in \mathbb{N}^{\mathbb{Z}} ; \sum_{k=1}^{L} \cap_{j}=N, n_{j}=1 \text { if } j<0 \text { and } n_{j}=0 \text { if } j \geq L\right\}
$$

with $L=[N / \varrho]$.

Suppose that we are given a Hamiltonian $H: Q_{N, \varrho} \mapsto \mathbb{R} \cup\{\infty\}$. For each $A \subset Q_{N, \varrho}$ we put

$$
Z(A)=Z_{N, \varrho, H}(A)=\sum_{\underline{n} \in A} \exp (-H(\underline{n})),
$$

where as usual $\exp (-\infty)=0$. We get a probability measure on the $\sigma$-algebra of all subsets of $Q_{N, \varrho}$ by letting

$$
P_{N, \varrho, H}(A)=Z_{N, \varrho, H}(A) / Z_{N, \varrho, H}\left(Q_{N, \varrho}\right)
$$

for each $A \subset Q_{N, \varrho}$. 
We want to show that, in the models we consider, there is a separation of phases at sufficiently low temperatures. Because of the choice of boundary conditions we expect to get a dense phase (liquid) to the left in $\Lambda$ and a dilute phase (vapour) to the right. The question is how far into $\Lambda$ the boundary conditions are felt. To make this more formal we need a definition. First, for each $\underline{n} \in Q_{N, \varrho}$ and $x, y \in \mathbb{Z}$, we put

$$
N(x, y)(\underline{n})=\sum_{i=x}^{y-1} n_{i} .
$$

Definition 1.1. Let $0 \leq \delta_{2}<\varrho<\delta_{1} \leq 1$ and $\omega \in(0,1)$. We will say that $t_{i}: Q_{N, \varrho} \rightarrow \mathbb{Z}^{+} \cap[0, L], i=1,2$, defines $a\left(\delta_{1}, \delta_{2}, \omega\right)$-phase separation if the following conditions are satisfied for each $\underline{n} \in Q_{N, \varrho}$ :

(i) if $x<t_{1}(\underline{n})$, then

$$
N\left(x, t_{1}\right) \geq \delta_{1}\left(t_{1}-x\right)
$$

(ii) if $x>t_{2}(\underline{n})$, then

$$
N\left(t_{2}, x\right) \leq \delta_{2}\left(x-t_{2}\right)
$$

(iii) Let $\left\{\varepsilon_{N}\right\}$ be any sequence tending to zero as $N \rightarrow \infty$. If $\left(x, x+\varepsilon_{N}^{-1} N^{\omega}\right) \subset\left(-\infty, t_{1}\right)$, then

$$
N\left(x, x+\varepsilon_{N}^{-1} N^{\omega}\right)(\underline{n}) \geq\left(\delta_{1}-o(1)\right) N^{\omega},
$$

and if $\left(x, x+\varepsilon_{N}^{-1} N^{\omega}\right) \subset\left(t_{2}, \infty\right)$, then

$$
\left(x, x+\varepsilon_{N}^{-1} N^{\omega}\right) \leq\left(\delta_{2}+o(1)\right) N^{\omega},
$$

for all sufficiently large $N$.

The interval $\left[0, t_{1}\right]$ should be thought of as containing the dense phase, density $\geq \delta_{1}$, and $\left[t_{2}, L\right]$ as containing the dilute phase, density $\leq \delta_{2}$. The third condition gives an upper bound on the size of "bubbles" in the dense phase and "droplets" in the dilute phase. The size of the largest bubble/droplet is less than essentially $N^{\omega}$.

By the choice of boundary conditions $t_{1}(\underline{n}) \equiv 0$ and $t_{2}(\underline{n}) \equiv L$ defines a $(1,0,0)$ phase separation. It is clear that we always have $t_{1}(\underline{n}) \leq \varrho L / \delta_{1}$.

That the boundary conditions are felt far into $\Lambda$ should mean that $t_{1}(\underline{n})$ and $t_{2}(\underline{n})$ are "close" for typical configurations. We can think of $\left(n_{t_{1}}, \ldots, n_{t_{2}}\right)$ as an interface in $\underline{n}$, and we will call

$$
\Delta(\underline{n})=t_{2}(\underline{n})-t_{1}(\underline{n})
$$

the thickness of the interface if $\underline{n}$. Note that $\Delta(\underline{n})$ depends on $\delta_{1}, \delta_{2}$ and $\omega$. In particular choosing a larger $\omega$, i.e. allowing larger bubbles/droplets, makes $\Delta(\underline{n})$ smaller. It is not clear what the right definition of the interface in a one-dimensional gas should be.

\subsection{The Continuous Model}

As above, let $L=[N / \varrho], \varrho \in \mathbb{R}^{+}$fixed. The configuration space is

$$
\Omega_{N, \varrho}=\left\{\underline{x} \in[0, L]^{N} ; x_{i}<x_{i+1}, i=1, \ldots, N-1\right\} .
$$


Also we introduce fixed particles at $x_{i}=i-1, i \leq 0$. On $\Omega_{N, \varrho}$ we consider the Hamiltonian

$$
H_{\phi}(\underline{x})=\frac{1}{2} \sum_{i, j=1, i \neq j}^{N} \phi\left(\left|x_{j}-x_{i}\right|\right)+\sum_{i=-\infty}^{0} \sum_{j=1}^{N} \phi\left(x_{j}-x_{i}\right)
$$

where

$$
\phi(r)=\frac{D}{r^{\gamma}}-\frac{C}{r^{2}}, \quad r>0,
$$

with $D, C \in \mathbb{R}^{+}$and $\gamma>2$.

For each Borel subset $B$ of $\Omega_{N, \varrho}$ we set

$$
Z^{c}(B)=\int_{B} \exp \left(-\beta H_{\phi}(\underline{x})\right) d x_{1} \ldots d x_{N},
$$

and the probability of $B$ is given by

$$
P_{N, \varrho, \phi}^{c}(B)=Z^{c}(B) / Z^{c}\left(\Omega_{N, \varrho}\right) .
$$

If we write $\phi(r)=\psi(r)-C / 2 r^{2}$, then $\phi(r)$ is increasing and concave, and $\psi(r)$ is increasing for $r \geq r_{0}$, where $r_{0}=[\max ((\gamma+1) \gamma D / 6 C, \gamma D / C)]^{1 /(\gamma-2)}$. After a change of variables (scaling) we can assume that $r_{0}=1$. The change of variables will modify the constants in the potential, and we will write (using the same notation)

$$
\phi(r)=\psi(r)-A / r^{2},
$$

where $\psi(r)=B / r^{\gamma}-A / r^{2}$. Now, $\phi(r)$ is strictly increasing and concave and $\psi(r)$ is increasing for $r \geq 1$.

To relate this model to the general lattice model above we need some further notation. Given $\underline{x} \in \Omega_{N, \varrho}$ we let $n_{i}(\underline{x})$ be the number of $x_{j}$ 's in $[i, i-1), i \in \mathbb{Z}$, and we write $\underline{n}(\underline{x})=\left(n_{i}(\underline{x})\right)_{i=-\infty}^{\infty}$. Clearly $\underline{n}(\underline{x}) \in Q_{N, \varrho}$ for all $\underline{x}$. Given $\underline{n} \in Q_{N, \varrho}$ we set

$$
\Omega(\underline{n})=\left\{\underline{x} \in \Omega_{N, \varrho} ; \underline{n}(\underline{x})=\underline{n}\right\},
$$

and for $A \subseteq Q_{N, \varrho}, \Omega(A)=\bigcup_{\underline{n} \in A} \Omega(\underline{n})$. Now, for any $A \subseteq Q_{N, \varrho}$,

$$
\begin{aligned}
Z^{c}(\Omega(A)) & =\sum_{\underline{n} \in A} \int_{\Omega(\underline{n})} \exp \left(-\beta H_{\phi}(\underline{x})\right) d^{N} x \\
& =\sum_{\underline{n} \in A} \exp \left(-H_{\phi}^{c}(\underline{n}, \beta)\right)
\end{aligned}
$$

where

$$
H_{\phi}^{c}(\underline{n}, \beta)=-\log \int_{\Omega(\underline{n})} \exp \left(-\beta H_{\phi}(\underline{x})\right) d^{N} x .
$$

Hence, for any $A \subseteq Q_{N, \varrho}$,

$$
P_{N, \varrho, \phi}^{c}(\Omega(A))=P_{N, \varrho, H_{\phi}^{c}}(A)
$$

which gives the relation between the continuous gas and the general lattice gas. We will write $\Delta(\underline{x})=\Delta(\underline{n}(\underline{x}))$. 
The main result for the continuous gas is the following theorem.

Theorem 1.2. There are positive constants $\beta^{*}, \eta_{2}, i=1, \ldots, 4$, which only depend on the parameters in $\phi$, such that if $\beta \geq \beta^{*}$, and $\varrho \in\left(\exp \left(-\eta_{1} \beta\right), 1 / 4\right)$, there is a $\left(1 / 2-\exp \left(-\eta_{1} \beta\right), \exp \left(-\eta_{1} \beta\right),\left(\eta_{2} \beta\right)^{-1}\right)$-phase separation, so that when $N^{\eta_{3} \exp \left(-\eta_{1} \beta\right)} \leq d \leq L$,

$$
P_{N, \varrho, \phi}^{c}\{\underline{x} \in \Omega ; \Delta(\underline{x}) \geq d\} \leq e^{\eta_{4} \beta^{2}} d^{-2}
$$

for all sufficiently large $N$.

This theorem shows that, for $\beta$ sufficiently large, with high probability the thickness of the interface is short on macroscopic length scales, and hence established the existence of a phase separations. The theorem will be proved in Sect. 4 .

The Helmholtz' free energy for the gas is defined by

$$
f(\varrho, \beta)=-\lim _{N \rightarrow \infty} \frac{\varrho}{\beta N} \log Z_{N, \varrho, \phi}^{c}\left(\Omega_{N, \varrho}\right),
$$

and the pressure is given by

$$
p(\mu, \beta)=\sup _{\varrho>0}(\mu \varrho / \beta-f(\varrho, \beta)),
$$

see [Ru1], Chap. 3.

Corollary 1.3. If $\beta \geq \beta^{*}$, there is a $\mu_{c}$ such that $\partial p / \partial \mu$ does not exist at $\left(\mu_{c}, \beta\right)$.

The proof of the corollary is sketched at the end of Sect. 5.

It is known that if the pressure is not differentiable as a function of $\mu$ at some point $\left(\beta, \mu_{c}\right)$, then there must exist several Gibbs states at this point, see [KY] Theorem 3.1(b).

\subsection{The $1 / r^{2}$-Lattice Gas}

In this section we consider an ordinary lattice gas, with at most one particle per lattice site, having a Hamiltonian which behaves like $1 / r^{2}$ for $r$ large. Let $Q_{N}^{*}=\left\{\underline{n} \in Q_{N, \varrho} ; 0 \leq n_{i} \leq 1, i \in \mathbb{Z}\right\}$ and set $H^{d}(\underline{n})=+\infty$ if $\underline{n} \in Q_{N, \varrho} \backslash Q_{N}^{*}$. If $\underline{n} \in Q_{N}^{*}$ set

$$
H^{d}(\underline{n})=-2 \beta \sum_{-\infty}^{\infty} \sum_{j=1}^{L} J(j-i) n_{i} n_{\jmath}
$$

where $J(r)$ satisfies

$$
\|J\|_{*}=\sum_{r=2}^{\infty} r\left|J(r)-1 / r^{2}\right|<\infty .
$$

With some extra work it is also possible to use the weaker condition $r^{2} J(r) \rightarrow 1$ as $r \rightarrow \infty$. Write $J_{1}=J(1)$. Note that we do not assume that $J(r) \geq 0$ for all $r \geq 1$.

By the substitution $s_{i}=2 n_{i}-1$, this model is related to the Ising model with Hamiltonian

$$
H^{I}(\underline{s})=-\frac{1}{2} \beta \sum_{i=-\infty}^{\infty} \sum_{j=1, i<j}^{L} J(j-i) s_{i} s_{j}+\beta h \sum_{i=1}^{L} s_{i}
$$


(with $+/-$ boundary conditions). Assume that $J(r) \geq 0$ and let $M\left(\beta, J_{1}\right)$ denote the spontaneous magnetization (using the usual + boundary conditions) as a function of $\beta$ and the nearest neighbour coupling $J_{1}$. Let $M^{*}(\beta)=\sup _{J_{1}} M\left(\beta, J_{1}\right)$ and $\beta^{*}=\sup \left\{\beta ; M^{*}(\beta)=0\right\}$. It is known that $\beta^{*} \leq 1$, [IN], i.e. if $\beta>1$ we can make $M\left(\beta, J_{1}\right)$ positive by choosing $J_{1}$ sufficiently large. The idea ot treating $J_{1}$ as an extra parameter was introduced in [ACCN].

We will now establish the corresponding result in the present setting.

Theorem 1.4. Assume that $J: \mathbb{Z}^{+} \rightarrow \mathbb{R}$ satisfies (1.4) and that the Hamiltonian is given by (1.3). Let $\xi>0, \kappa \in(0,1)$ and $\lambda>2$ be given. Suppose that $\varrho \in(1 / \lambda, 1-1 / \lambda)$. Put $\mu^{2}=1-4 / \lambda+1 / \lambda^{2}$ and assume that $\beta \mu^{2}>1+\xi / 2$. There is a constant $J_{1}^{*}$ such that if $J_{1}>J_{1}^{*}$, then there is a $\left(1-1 / \lambda, 1 / \lambda,(1+\xi)^{-1}\right)$ phase separation so that for all $d \geq 1$,

$$
P_{N, \varrho, H^{d}}\left\{\underline{n} \in Q_{N, \varrho} ; \Delta(\underline{n}) \geq d\right\} \leq C d^{-\psi}
$$

where $C$ is a constant, $N$ is sufficiently large and

$$
\psi=\frac{2\left(\beta \mu^{2}-1-\xi / 2\right)(1-\kappa)}{1+\xi} .
$$

The proof will be given in Sect. 3 .

Let us give some heuristic comments. Inequality (1.6) implies that in the corresponding Ising model, the spontaneous magnetization $M=M\left(\beta, J_{1}\right)>0$. We have the inequality $M \geq 1-2 / \lambda>\mu$, and for given $\beta, J_{1}$, the largest possible $\lambda$ should be $\lambda=2(1-M)^{-1}$. Picking $\kappa$ and $\xi$ small, we see that the exponent $\psi$ in (1.6) is approximately $2\left(\beta M^{2}-1\right)$. This should be compared with the known fact [IN], that the $1 / r^{2}$-Ising model has an intermediate phase, where the correlation function $\left|\left\langle s_{0} s_{x}\right\rangle-M^{2}\right| \sim|x|^{-\theta}$, with $\theta \leq 2\left(\beta M^{2}-1\right)$ and $\theta \rightarrow 2(\beta-1)$ as $J_{1} \rightarrow \infty$; it is conjectured that $\theta=2\left(\beta M^{2}-1\right)$ in the intermediate phase [IN].

The largest possible $\xi$ we can choose, keeping $\psi$ positive, is $\xi=2\left(\beta \mu^{2}-1\right)-\varepsilon / 2$, $\varepsilon>0$ arbitrary. Hence the best estimate we can get on the size of the largest bubbles/droplets is that they are $\leq N^{\left[2 \beta \mu^{2}-1-\varepsilon / 2\right]^{-1}}$, which is close to $N^{(1+\theta)^{-1}}$. As the critical line is approached we expect that $\theta \rightarrow 0$, and then the upper bound on the size of maximal bubbles/droplets approaches $O(N)$.

Define

$$
\beta_{c}\left(J_{1}\right)=\sup \left\{\beta ; M\left(\beta, J_{1}\right)=0\right\} .
$$

Corollary 1.5. There is a constant $C_{1}$ such that if $J_{1}>C_{1}$, then

$$
\beta_{c}\left(J_{1}\right)-1 \leq e^{2 C_{1} / 5} e^{-2 J_{1} / 5} .
$$

Proof. See Sect. 3.

Remark 1.6. Going through the proof in Sect. 3 and estimating the constants numerically, it is seen that $C_{1} \approx 8+8\|J\|_{*}$ suffices.

Remark 1.7. It is conjectured that the critical line $\beta=\beta_{c}\left(J_{1}\right)$ is given by $\beta M^{2}\left(\beta, J_{1}\right)=1$, and it seems reasonable that $M\left(\beta_{c}\left(J_{1}\right)-, J_{1}\right)=1-O\left(\exp \left(-O\left(J_{1}\right)\right)\right)$. This gives $\beta_{c}\left(J_{1}\right)-1=O\left(\exp \left(-O\left(J_{1}\right)\right)\right)$, so the exponential dependence on $J_{1}$ in (1.8) should be correct.

Remark 1.8. The existence of a phase separation can also be proved when $J(r)$ is arbitrary in $\mathbb{R} \cup\{\infty\}$ for $r<r_{0}$ and $J(r), r \geq r_{0}$, is decreasing and $r^{2} J(r) \rightarrow J^{+}>0$ as $r \rightarrow \infty$. See Remark 4.2. 


\section{The Energy-Entropy Argument}

\subsection{Partitions and the Rearrangement Procedure}

In this section we will develop the necessary combinatorics and carry out the energy-entropy argument, under suitable assumptions on the Hamiltonian $H: Q_{N, \varrho} \rightarrow$ $\mathbb{R} \cup\{\infty\}$, for the general one-dimensional lattice as defined in Sect. 1 .

We will use the notation introduced in Sect. 1 and $|M|$ will denote the cardinality of an arbitrary set $M$. Define

$$
\mathscr{P}=\left\{(\underline{n}, \gamma) ; \underline{n} \in Q_{N, \varrho}, \gamma \subseteq[0, L] \cap \mathbb{Z}, \text { with }|\gamma| \text { odd }\right\} .
$$

For $\Gamma=(\underline{n}, \gamma) \in \mathscr{P}$ we will write $\pi_{1} \Gamma=\underline{n}$ and $\pi_{2} \Gamma=\gamma$. In the ordered pair $(\underline{n}, \gamma), \gamma$ is called a partition and it defines a partition of the configuration $\underline{n}$ into blocks. Given $\underline{n}$ and $a, a^{\prime} \in[0, L] \cap \mathbb{Z}$, the block $\left\langle a, a^{\prime}\right\rangle=\left(n_{a}, \ldots, n_{a^{\prime}-1}\right)$. If $A^{\prime}=\left\langle b, b^{\prime}\right\rangle$ is another block, then the block obtained by joining $A$ and $A^{\prime}$ is $A A^{\prime}=\left(n_{a}, \ldots, n_{a^{\prime}-1}, n_{b}, \ldots, n_{b^{\prime}-1}\right)$. Let $\Gamma=(\underline{n}, \gamma) \in \mathscr{P}$ be given with $\gamma=\left\{b_{1}, a_{2}, b_{2}, \ldots, a_{s}, b_{s}\right\}$, where $b_{1}<a_{2}<\ldots<a_{s}<b_{s}$. This gives a partition of $\underline{n}$ into blocks $A_{1}, B_{1}, \ldots, A_{s}, B_{s}$, where $A_{\jmath}=\left\langle a_{\jmath}, b_{\jmath}\right\rangle$ and $B_{\jmath}=\left\langle b_{j}, a_{\jmath+1}\right\rangle$, $j=1, \ldots, s$. Here $a_{1}=-\infty$ and $a_{s+1}=\infty$. We will call $A_{1}, \ldots, A_{s}$ o-blocks, $B_{1}, \ldots, B_{s}$ e-blocks and we will say that these are the blocks in $\Gamma$.

Let $C=\left\langle c, c^{\prime}\right\rangle$ be a block in $\Gamma \in \mathscr{P}$ and define the length $|C|$ of $C$ to be $c^{\prime}-c$. The weight $w(C)$ of $C$ is defined to be

$$
w(C)=\sum_{i=c}^{c^{\prime}-1} \max \left(1, n_{i}\right) .
$$

From the definitions it is clear that

$$
|C| \leq w(C)
$$

for every block $C$ in $\Gamma$. If we restrict ourselves to configurations with $n_{i} \leq 1$ for all $i$, as in a standard lattice gas, then equality holds in (2.1).

We now turn to the definition of the partitions that we will work with. Assume that every $\underline{n} \in Q_{N, \varrho}$ has been assigned a basic partition $\gamma^{(0)}(\underline{n})$, i.e. a subset $\gamma^{(0)}(\underline{n}) \subseteq\{1, \ldots, L\}$ with an odd number of elements. Let $\lambda$ and $\xi$ be given positive parameters and for $j \geq 1$ set

$$
u_{\jmath}=2^{\jmath-1} ; \quad v_{\jmath}=C(\xi) \lambda u_{\jmath}^{1+\xi}
$$

where

$$
C(\xi)=2^{\xi}\left(2^{\xi}-1\right)^{-1}
$$

We can now iteratively define partitions $\gamma^{(k)}(\underline{n})$ in the following way (compare [Jo1] p. 45). Suppose that $\gamma^{(k-1)}(\underline{n})$ has been defined. Consider all $e$-blocks in $\left(\underline{n}, \gamma^{(k-1)}(\underline{n})\right)$ of length $\geq v_{k}, B_{j_{1}}, \ldots, B_{j_{p}}, p \geq 1$. If the o-blocks in $\left(\underline{n}, \gamma^{(k)}(\underline{n})\right)$ between $B_{j_{r}}=\left\langle b_{\jmath_{r}}, a_{\jmath_{r}+1}\right\rangle$ and $B_{\jmath_{r+1}}=\left\langle b_{\jmath_{r+1}}, a_{\jmath_{r+1}+1}\right\rangle$ have total weight $\leq u_{k}$ we set $\eta_{r}=\left[a_{j_{r}+1}, b_{j_{r+1}}\right] \cap \gamma^{(k-1)}(\underline{n})$; if the $o$-blocks have total weight $>u_{k}$ we set $\eta_{r}=\emptyset$. Do this for $r=1, \ldots, p-1$; if $p=1$ there are not $\eta_{r}$ 's. In the same way we define $\lambda_{1}, \ldots, \lambda_{q-1}$ with $e$ - and $o$-blocks interchanged. Let

$$
\gamma^{(k)}(\underline{n})=\gamma^{(k-1)}(\underline{n}) \backslash\left(\eta_{1} \cup \ldots \cup \eta_{p-1} \cup \lambda_{1} \cup \ldots \cup \lambda_{q-1}\right) .
$$


Note that we remove an even number of elements from $\gamma^{(k-1)}(\underline{n})$, so $\left|\gamma^{(k)}(\underline{n})\right|$ is odd. Let

$$
\gamma_{1}(\underline{n})=\bigcap_{k \geq 0} \gamma^{(k)}(\underline{n})
$$

and, for each $\underline{n} \in Q_{N, \varrho}$, define $\Gamma_{1}(\underline{n})=\left(\underline{n}, \gamma_{1}(\underline{n})\right)$.

It follows from the construction that the following lemma holds (see [Jo1], p. 45 for more details).

Lemma 2.1. Consider two o-(e-)blocks of length $\geq v_{k}$ in $\Gamma_{1}(\underline{n}), \underline{n} \in Q_{N, \varrho}$. Then the total weight of the e-(o-)blocks between them is $>u_{k}, k \geq 1$.

The choice of the basic partition $\gamma^{(0)}(\underline{n})$ should be such that $o$-blocks have a higher density of particles than the $e$-blocks. Before stating the precise definition we need some notation. Given $\Gamma \in \mathscr{P}$ with $\pi_{1} \Gamma=\underline{n}, x, y \in \mathbb{Z}$, we write

$$
N(x, y)=\sum_{i=x}^{y-1} n_{i}
$$

and

$$
\nu(x, y)=\sum_{i=x}^{y-1} \min \left(1, n_{i}\right) .
$$

Definition 2.2. We will say that $\Gamma \in \mathscr{P}$ satisfies the $\left(\delta_{1}, \delta_{2}\right)$-density property, $0 \leq$ $\delta_{2}<\delta_{1} \leq 1$, if

(1) for each o-block $A=\left\langle a, a^{\prime}\right\rangle$ in $\Gamma$, and all $x \in\left[a, a^{\prime}-1\right]$,

(i) $\nu(a, x) \geq \delta_{1}(x-a)$,

(ii) $\nu\left(x, a^{\prime}\right) \geq \delta_{1}\left(a^{\prime}-x-1\right)$;

(2) for each e-block $B=\left\langle b, b^{\prime}\right\rangle$ in $\Gamma$, and all $x \in\left[b, b^{\prime}\right]$,

(i) $N(b, x) \leq \delta_{2}(x-b)$,

(ii) $N\left(x, b^{\prime}\right) \leq \delta_{2}\left(b^{\prime}-x-1\right)$,

(iii) $|B| \leq w(B) \leq \delta_{1}^{-1}|B|$.

We can now prove

Lemma 2.3. If $\left(\underline{n}, \gamma^{(0)}(\underline{n})\right), \underline{n} \in Q_{N, \varrho}$, satisfies the $\left(\delta_{1}^{(0)}, \delta_{2}^{(0)}\right)$-density property, then $\Gamma_{1}(\underline{n})$ satisfies the $\left(\delta_{1}, \delta_{2}\right)$-density property with

$$
\delta_{1}=\delta_{1}^{(0)}-1 / \lambda, \quad \delta_{2}=\delta_{2}^{(0)}+1 / \lambda
$$

where $\lambda$ is the parameter in (2.2). 2.2 .

Note that we assume that $\lambda$ is chosen so that $\delta_{1}>\delta_{2}$ are required in Definition

Proof. See the end of this section.

We will now define the rearrangement operations which will be used to transform a configuration into the corresponding condensed configuration. Given $j \geq 1$ the $\left(j^{\text {th }}\right.$ order) elementary rearrangement operation $S_{j}: \mathscr{P} \rightarrow \mathscr{P}$ is defined as follows. 
(The present definition differs from the one used in [Jo1].) Let $\Gamma \in \mathscr{P}$ and let $C^{(1)}, \ldots, C^{(t)}$ be the blocks in $\Gamma$. Set

$$
W(\Gamma)=\min _{1 \leq i \leq t} w\left(C^{(i)}\right)
$$

i.e. the smallest weight of a block in $\Gamma$. If $W(\Gamma) \geq u_{\jmath+1}$, we set $S_{j} \Gamma=\Gamma$. Otherwise let $C^{(k)}$ be the first block (counting from the left) of weight $<u_{j+1} . S_{\jmath} \Gamma$ is obtained from $\Gamma$ by letting $C^{(k)}$ and $C^{(k+1)}$ change place, except when $k=t-1$, in which case we let $C^{(t-2)}$ and $C^{(t-1)}$ change place. More formally, that $C^{(k)}=\left\langle c_{k}, c_{k+1}\right\rangle$ and $C^{(k+1)}=\left\langle c_{k+1}, c_{k+2}\right\rangle$ change place means that we set

$$
\begin{aligned}
& \pi_{1} S_{\jmath} \Gamma=C^{(1)} \ldots C^{(k-1)} C^{(k+1)} C^{(k)} C^{(k+2)} \ldots C^{(t)}, \\
& \pi_{2} S_{j} \Gamma=\left(\pi_{2} \Gamma \backslash\left\{c_{k}, c_{k+1}, c_{k+2}\right\}\right) \cup\left\{c_{k}+c_{k+2}-c_{k+1}\right\} .
\end{aligned}
$$

The points $c_{k}$ and $c_{k+2}$ will be called "old" partition points. Note that if we specify $c_{k}$ and $c_{k+2}$ we can recover $\Gamma$ from $S_{j} \Gamma$ uniquely, so the fact that different choices of $c_{k}$ and $c_{k+2}$ gives different $\Gamma$ given $S_{j} \Gamma$ means that we have a decrease in entropy. The "old" partition points always come in pairs and we will say that they constitute a pair of "old" partition points, one of them lies in an $o$-block and the other in an $e$-block.

Set

$$
\mathscr{P}_{1}=\left\{\Gamma_{1}(\underline{n}) ; \underline{n} \in Q_{N, \varrho}\right\} \text {. }
$$

We want to define $\mathscr{P}_{j} \subseteq \mathscr{P}, j \geq 2$. Assume that $\mathscr{P}_{j}$ has been defined. For $\Gamma \in \mathscr{P}_{j}$ we let

$$
k_{j}(\Gamma)=\min \left\{k \in N ; W\left(S_{j}^{k} \Gamma\right) \geq u_{\jmath+1}\right\},
$$

Here $S_{j}^{k}=S_{j} \circ \ldots \circ S_{\jmath}$ ( $k$ times). The map $R_{j}: \mathscr{P}_{j} \rightarrow \mathscr{P}$ is now defined by $R_{\jmath} \Gamma=S_{j}^{k_{j}(\Gamma)} \Gamma$ and we set $\mathscr{P}_{\jmath+1}=R_{\jmath}\left(\mathscr{P}_{j}\right)$. It is clear from the definition that

$$
\Gamma \in \mathscr{P}_{j} \Rightarrow W(\Gamma) \geq u_{j}, \quad j \geq 1 .
$$

We are now in position to define the condensed configuration corresponding to a given configuration. Let

$$
p=1+\left[\log _{2}\left(\frac{1}{\varrho}+1\right) N\right]
$$

Then

$$
\Gamma \in \mathscr{P}_{p+1} \Rightarrow\left|\pi_{2} \Gamma\right|=1 .
$$

To see this note that if $\left|\pi_{2} \Gamma\right|>1$, then $W(\Gamma)<L+N \leq N(1 / \varrho+1)$, but from (2.6), $W(\Gamma) \geq 2^{p}>N(1 / \varrho+1)$, and we get a contradiction. Define the maps $\mathscr{B}_{j}: \mathscr{P}_{1} \rightarrow \mathscr{P}_{j+1}, j \geq 1$, by $\mathscr{B}_{j}=R_{j} \circ R_{j-1} \circ \ldots \circ R_{1}$. It follows from (2.8), that if $A_{1}, B_{1}, \ldots, A_{s}, B_{S}$ are the blocks in $\Gamma_{1}(\underline{n})$, so that $\underline{n}=A_{1} B_{1} \ldots A_{s} B_{s}$, then

$$
\mathscr{B}(\underline{n}) \doteq \pi_{1} \mathscr{B}_{p} \Gamma_{1}(\underline{n})=A_{1} A_{2} \ldots A_{s} B_{1} B_{2} \ldots B_{s} .
$$

For a configuration $\underline{n} \in Q_{N, \varrho}, \mathscr{B}(\underline{n})$ is the corresponding condensed configuration. 


\subsection{The Entropy Estimate}

Before stating the basic entropy estimate we need some further notation. Write $\Gamma_{j}(\underline{n})=\mathscr{R}_{j-1} \Gamma_{1}(\underline{n})$ and for a given $A \subseteq Q_{N, \varrho}, \mathscr{P}_{j}(A)=\left\{\Gamma_{j}(\underline{n}) ; \underline{n} \in A\right\}$. Note that $\mathscr{P}_{j}(A)=R_{\jmath-1} \mathscr{P}_{j-1}(A)$. Also

$$
Z(A)=\sum_{\Gamma \in \mathscr{P _ { 1 }}(A)} e^{-H\left(\pi_{1} \Gamma\right)}
$$

since the correspondence $\underline{n} \mapsto \Gamma_{1}(\underline{n})$ is one-to-one. Given $A \subseteq Q_{N, \varrho}, \underline{k}=$ $\left(k_{1}, \ldots, k_{p}\right) \in N^{p}$ and $\Gamma_{0} \in \mathscr{P}_{p+1}(A)$, we write

$$
\mathscr{P}_{1}\left(A, \Gamma_{0}, \underline{k}\right)=\left\{\Gamma \in \mathscr{P}_{1}(A) ; \mathscr{B}_{p} \Gamma=\Gamma_{0}, \underline{k}(\Gamma)=\underline{k}\right\},
$$

where $\underline{k}(\Gamma)=\left(k_{1}(\Gamma), \ldots, k_{p}(\Gamma)\right)$. We will also write $k_{\jmath}(\underline{n})=k_{j}\left(\Gamma_{j}(\underline{n})\right), j=$ $1, \ldots, p$, and $\underline{k}(\underline{n})=\left(k_{1}(\underline{n}), \ldots, k_{p}(\underline{n})\right)$. Also set

$$
\mathscr{P}_{1}(A, \underline{k})=\bigcup_{\Gamma_{0} \in \mathscr{P}_{p+1}(A)} \mathscr{P}_{1}\left(A, \Gamma_{0}, \underline{k}\right) .
$$

The entropy change, as well as the energy change, in the rearrangement procedure will be estimated using the quantities

$$
s(\underline{k})=\sum_{j=1}^{p} k_{j}
$$

and

$$
\sigma(\underline{k})=\sum_{j=1}^{p} j k_{j}
$$

Note that $1+s(\underline{k}(\underline{n}))=s=$ the number of $o-(e-)$ blocks in $\Gamma_{1}(\underline{n})$.

We can now prove

Lemma 2.6 (The entropy lemma). Let $A \subseteq Q_{N, \varrho}, \Gamma_{0} \in \mathscr{P}_{p+1}(A)$ and $\underline{k} \in \mathbb{N}^{p}$ be given. Then

$$
\left|\mathscr{P}_{1}\left(A, \Gamma_{0}, \underline{k}\right)\right| \leq \frac{(s(\underline{k})) !}{\prod_{j=1}^{p} k_{j} !} e^{c_{1} s(\underline{k})+c_{2} \sigma(\underline{k})}
$$

where

$$
\begin{aligned}
& c_{1}=\log \left[5 C(\xi)^{2} \lambda 2^{1+\xi}\right], \\
& c_{2}=(2+\xi) \log 2 .
\end{aligned}
$$

Proof. Define for $2 \leq j \leq p+1$,

$$
\mathscr{P}_{j}\left(A, \Gamma_{0}, \underline{k}\right)=\left\{\mathscr{R}_{j-1} \Gamma ; \Gamma \in \mathscr{P}_{1}\left(A, \Gamma_{0}, \underline{k}\right)\right\} .
$$

The first step is to prove

$$
\left|\left\{\Gamma^{\prime} \in \mathscr{P}_{j}\left(A, \Gamma_{0}, \underline{k}\right) ; R_{\jmath} \Gamma^{\prime}=\Gamma\right\}\right| \leq\left(\begin{array}{c}
k_{p}+\ldots+k_{j} \\
k_{\jmath}
\end{array}\right) e^{c_{1} k_{\jmath}+c_{2} j k_{\jmath}}
$$

for each $\Gamma \in \mathscr{P}_{j+1}\left(A, \Gamma_{0}, \underline{k}\right)$, with $c_{1}, c_{2}$ given by (2.10). 
From the definition of the rearrangement it is clear that each $o-(e-)$ block $C$ in $\Gamma \in \mathscr{P}_{\jmath+1}$ is of the form $C=C_{\imath} C_{\imath+1} \ldots C_{\jmath}$ for some $o$-(e-)blocks $C_{\imath}, \ldots, C_{\jmath}$ in $\Gamma^{\prime}$ if $\Gamma=R_{\jmath} \Gamma^{\prime}, \Gamma^{\prime} \in \mathscr{P}_{\jmath}$. To reconstruct $\Gamma^{\prime}$ from $\Gamma$ uniquely we have to specify the positions of $2 k_{\jmath}\left(\Gamma^{\prime}\right)$ old partition points. As explained above these occur in pairs, with one of the points in the pair, called the old o-point, lying in an o-block in $\Gamma$, and the other, called the old e-point, lying in an $e$-block in $\Gamma$.

Claim 2.7. Given that the old o-point in a pair of old partition points lies in a certain o-block in $\Gamma$, there are $\leq \exp \left(c_{1}+c_{2} j\right)$ possibilities for the pair, where $c_{1}, c_{2}$ are given by (2.10).

We postpone the proof to the end of the section.

If $\Gamma^{\prime} \in \mathscr{P}_{\jmath}\left(A, \Gamma_{0}, \underline{k}\right)$ and $\Gamma=R_{\jmath} \Gamma^{\prime}$, then $\left|\pi_{2} \Gamma^{\prime}\right|-\left|\pi_{2} \Gamma\right|=2 k_{\jmath}$, and using this repeatedly we obtain $\left|\pi_{2} \Gamma\right|=2\left(k_{p}+\ldots+k_{j+1}\right)+1$, since $\left|\pi_{2} \Gamma_{0}\right|=1$. The number of $o$-blocks in $\Gamma$ is $\left(\left|\pi_{2} \Gamma\right|+1\right) / 2=k_{p}+\ldots+k_{\jmath+1}+1$. If we have $k_{j}$ pairs of old partition points there are less than or equal to

$$
\left(\begin{array}{c}
k_{p}+\ldots+k_{\jmath+1}+1+k_{\jmath}-1 \\
k_{\jmath}
\end{array}\right)=\left(\begin{array}{c}
k_{p}+\ldots+k_{\jmath} \\
k_{\jmath}
\end{array}\right)
$$

ways to distribute the $k_{\jmath}$ old partition points among the $o$-blocks in $\Gamma$. This together with Claim 2.7 gives (2.11).

Now,

$$
\mathscr{P}_{\jmath}\left(A, \Gamma_{0}, \underline{k}\right)=\bigcup_{\mathscr{P}_{\jmath+1}\left(A, \Gamma_{0}, \underline{k}\right)}\left\{\Gamma^{\prime} \in \mathscr{P}_{\jmath}\left(A, \Gamma_{0}, \underline{k}\right) ; R_{\jmath} \Gamma^{\prime}=\Gamma\right\},
$$

and consequently using (2.11),

$$
\frac{\left|\mathscr{P}_{j}\left(A, \Gamma_{0}, \underline{k}\right)\right|}{\left|\mathscr{P}_{\jmath+1}\left(A, \Gamma_{0}, \underline{k}\right)\right|} \leq \frac{\left(k_{p}+\ldots+k_{j}\right) !}{\left(k_{p}+\ldots+k_{\jmath+1}\right) ! k_{\jmath} !} e^{c_{1 \jmath}+c_{2} j k_{\jmath}} .
$$

Since $\left|\mathscr{P}_{p+1}\left(A, \Gamma_{0}, \underline{k}\right)\right|=\left|\left\{\Gamma_{0}\right\}\right|=1$ repeated use of this estimate completes the proof of the lemma.

\subsection{The Energy-Entropy Balance}

Let us now define the $\left(\delta_{1}, \delta_{2}, \omega\right)$-phase separation that we will use. Consider $\underline{n} \in Q_{N, \varrho}$ and let

$$
\pi_{2} \Gamma_{1}(\underline{n})=\left\{b_{1}(\underline{n}), a_{2}(\underline{n}), b_{2}(\underline{n}), \ldots, a_{s}(\underline{n}), b_{s}(\underline{n})\right\}, \quad b_{1}(\underline{n})<a_{2}(\underline{n})<\ldots<b_{2}(\underline{n}) .
$$

Put

$$
t_{1}(\underline{n})=b_{1}(\underline{n}), \quad t_{2}(\underline{n})=b_{s}(\underline{n}),
$$

so that $t_{1}(\underline{n}), t_{2}(\underline{n})$ are the rigth and left endpoints of the first and last $o$-blocks respectively.

Lemma 2.8. $t_{1}(\underline{n}), t_{2}(\underline{n})$ defined by (2.12) defines a $\left(\delta_{1}, \delta_{2}, \omega\right)$-phase separation with $\delta_{1}=\delta_{1}^{(0)}-1 / \lambda, \delta_{2}=\delta_{2}^{(0)}+1 / \lambda$ and $\omega=(1+\xi)^{-1}$.

Proof. See the end of this section. 
Lemma 2.9. For each $\underline{n} \in Q_{N, \varrho}$,

$$
\sigma(\underline{k}(\underline{n})) \geq \frac{\log \Delta(\underline{n})-(1+\xi) \log 2-c_{1}}{(1+\xi) \log 2}+1
$$

with $c_{1}$ given by (2.10).

Proof. See the end of the section.

We now come to the main result for the general lattice gas.

Proposition 2.10. Let the density $\varrho \in \mathbb{R}^{+}$, the Hamiltonian $H: Q_{N, \varrho} \rightarrow R \cup\{\infty\}$, a basic partition $\gamma^{(0)}(\underline{n})$, satisfying the $\left(\delta_{1}^{(0)}, \delta_{2}^{(0)}\right)$-density property, for each $\underline{n} \in Q_{N, \varrho}$ and a subset $Q_{N}^{*}$ of $Q_{N, \varrho}$ be given. Assume that $\xi$ and $\lambda$ in (2.2) and $D, D \geq$ $2^{1+\xi} \exp \left(c_{1}\right)$, can be chosen so that $\delta_{1}=\delta_{1}^{(0)}-1 / \lambda>\delta_{2}^{(0)}+1 / \lambda=\delta_{2}$, and

$$
H(\underline{n})-H(\mathscr{R} \underline{n}) \geq d_{1} s(\underline{k}(\underline{n}))+d_{2} \sigma(\underline{k}(\underline{n})),
$$

for any $\underline{n} \in Q_{N, \varrho} \backslash Q_{N}^{*}$ satisfying $\Delta(\underline{n}) \geq D$, where the constants $d_{1}, d_{2}$ satisfy

$$
\begin{aligned}
& K_{1}=d_{1}-c_{1}+\log \left(\exp \left(\kappa\left(d_{2}-c_{2}\right)\right)-1\right)>0 \\
& K_{2}=d_{2}-c_{2}>0
\end{aligned}
$$

for some $\kappa \in(0,1)$ with $c_{1}, c_{2}$ given by (2.10). Then for $d \geq D$,

$$
P_{N, \varrho, H}\left\{\underline{n} \in Q_{N, \varrho} ; \Delta(\underline{n}) \geq d\right\} \leq P_{N, \varrho, H}\left(Q_{N}^{*}\right)+C d^{-\psi},
$$

where

$$
\begin{aligned}
\psi & =\frac{(1-\kappa)\left(d_{2}-c_{2}\right)}{(1+\xi) \log 2} \\
C & =\exp \left(\psi\left((1+\xi) \log 2+c_{1}\right)\right)\left(\exp K_{1}-1\right)^{-1}\left(\exp K_{2}-1\right)^{-1}
\end{aligned}
$$

Proof. Let $X=\left\{\underline{n} \in Q_{N, \varrho} \backslash Q_{N}^{*} ; \sigma(\underline{k}(\underline{n})) \geq \sigma_{0}\right\}$, where $\sigma_{0} \geq 1$. If we can show that

$$
P_{N, \varrho, H}(X) \leq C^{\prime} \exp \left(-K_{2}(1-\kappa) \sigma_{0}\right)
$$

then combining this with (2.13) we obtain (2.16); $C^{\prime}=\left(\exp K_{1}-1\right)^{-1}\left(\exp K_{2}-1\right)^{-1}$. Note that $\Delta(\underline{n}) \geq 2^{1+\xi} \exp c_{1}$ implies $\sigma(\underline{k}(\underline{n})) \geq 1$.

By (2.9),

$$
\begin{aligned}
Z(X) & =\sum_{\Gamma^{\prime} \in \mathscr{P}_{1}(X)} e^{-H\left(\pi_{1} \Gamma^{\prime}\right)} \\
& =\sum_{\Gamma \in \mathscr{P}_{p+1}(X)} e^{-H\left(\pi_{1} \Gamma\right)} \sum_{\Gamma^{\prime} \in \mathscr{P}_{1}(X) ; \mathscr{R}_{p} \Gamma^{\prime}=\Gamma} \\
& =\sum_{\Gamma \in \mathscr{P}_{p+1}(X)} e^{-H\left(\pi_{1} \Gamma\right)} \sum_{s=1}^{\infty} \sum_{\sigma=\sigma_{0}}^{\infty} \sum_{\underline{k} \in K_{s, \sigma}} \sum_{\Gamma^{\prime} \in \mathscr{P}_{1}(X ; \Gamma, \underline{k})} e^{-\left(H\left(\pi_{1} \Gamma^{\prime}\right)-H\left(\pi_{1} \mathscr{B}_{p} \Gamma^{\prime}\right)\right)},
\end{aligned}
$$


where $K_{s, \sigma}=\{\underline{k} ; s(\underline{k})=s$ and $\sigma(\underline{k})=\sigma\}$. We now use Lemma 2.6 and the estimate (2.14) to get

$$
\begin{aligned}
Z(X) \leq & \sum_{\Gamma \in \mathscr{P}_{p+1}(X)} e^{-H\left(\pi_{1} \Gamma\right)} \sum_{s=1}^{\infty} e^{-b_{1} s} \sum_{\sigma=\sigma_{0}}^{\infty} e^{-(1-\kappa) b_{2} \sigma} \sum_{\underline{k} \in K_{s, \sigma}} \\
& \times e^{-\kappa b_{2} \sigma} \frac{s !}{\prod_{j=1}^{p} k_{j} !}
\end{aligned}
$$

where $b_{i}=d_{i}-c_{\imath}, i=1,2$. The multinomial theorem yields

$$
\begin{aligned}
\sum_{\underline{k} \in K_{s, \sigma}} e^{-\kappa b_{2} \sigma} \frac{s !}{\prod_{j=1}^{p} k_{\jmath} !} & =\sum_{\underline{k} \in K_{s, \sigma}} \frac{s !}{\prod_{j=1}^{p} k_{j} !} \prod_{j=1}^{p}\left(e^{-\kappa b_{2} j}\right)^{k_{j}} \\
& \leq\left(\sum_{j=1}^{p} e^{-\kappa b_{2} \jmath}\right)^{s} \leq\left(e^{\kappa b_{2}}-1\right)^{-s}=e^{b_{3} s},
\end{aligned}
$$

where $b_{3}=-\log \left(e^{\kappa b_{2}}-1\right)$. Inserting this in (2.19) we see that

$$
\begin{aligned}
Z(X) & =\sum_{\Gamma \in \mathscr{P}_{p+1}(X)} e^{-H\left(\pi_{1} \Gamma\right)} \sum_{s=1}^{\infty} e^{-\left(b_{1}-b_{3}\right) s} \sum_{\sigma=\sigma_{0}}^{\infty} e^{-(1-\kappa) b_{2} \sigma} \\
& \leq C e^{-(1-\kappa) b_{2}\left(\sigma_{0}-1\right)} \sum_{\Gamma \in \mathscr{P}_{p+1}(X)} e^{-H\left(\pi_{1} \Gamma\right)}
\end{aligned}
$$

since $b_{2}>0$ and $b_{1}-b_{3}>0$ by $(2.15)$.

To complete the proof of (2.18) we need to show that

$$
\sum_{\Gamma \in \mathscr{P}_{p+1}(X)} e^{-H\left(\pi_{1} \Gamma\right)} \leq Z\left(Q_{n, \varrho}\right) .
$$

This follows if we can prove that for any $\Gamma, \Gamma^{\prime} \in \mathscr{P}_{p+1}(X), \pi_{1} \Gamma=\pi_{1} \Gamma^{\prime} \Rightarrow \pi_{2} \Gamma=$ $\pi_{2} \Gamma^{\prime}$. Assume that $\pi_{1} \Gamma=\pi_{1} \Gamma^{\prime}$ but $\pi_{2} \Gamma=\{x\} \neq\{y\}=\pi_{2} \Gamma^{\prime}$, where we can suppose that $x<y$. It follows from the assumptions that both $\Gamma$ and $\Gamma^{\prime}$ satisfy the $\left(\delta_{1}, \delta_{2}\right)$-density property. Since $\pi_{1} \Gamma=\pi_{1} \Gamma^{\prime}, N(x, y)(\Gamma)=N(x, y)\left(\Gamma^{\prime}\right)$. Now, by the density property for $\Gamma, N(x, y)(\Gamma) \leq \delta_{2}(y-x)$, and by the density property for $\Gamma^{\prime}, N(x, y)\left(\Gamma^{\prime}\right) \geq \delta_{1}(y-x)$. Since $\delta_{1}>\delta_{2}$, we get a contradiction.

This completes the proof of Proposition 2.5.

\section{Proof of Some Lemmas}

Proof of Lemma 2.3. We proceed inductively. Define

$$
\delta_{1}^{(k)}=\delta_{1}^{(0)} \prod_{i=1}^{k}\left(1-u_{i} / v_{\imath}\right) ; \quad \delta_{2}^{(k)}=\delta_{2}^{(0)}+\sum_{i=1}^{k} u_{i} / v_{\imath}
$$

for $k \geq 1$. Suppose that $\left.\underline{n}, \gamma^{(k-1)}(\underline{n})\right)$ satisfies the $\left(\delta_{1}^{(k-1)}, \delta_{2}^{(k-1)}\right)$-density property. Consider an $o$-block $\left\langle c, c^{\prime}\right\rangle$ in $\left(\underline{n}, \gamma^{(k)}(\underline{n})\right)$ and let $l$ and $l^{\prime}$ be the weight and length, 
respectively, of the $e$-blocks (or parts of $e$-blocks) in $\left(\underline{n}, \gamma^{(k-1)}(\underline{n})\right.$ ) between $c$ and $c+t<c^{\prime}$. The construction of $\gamma^{(k)}(\underline{n})$ from $\gamma^{(k-1)}(\underline{n})$ above shows that $t-l^{\prime} \geq v_{k} l / u_{k}$, which gives $l \leq u_{k} t / v_{k}$, and hence $l^{\prime} \leq u_{k} t / v_{k}$ since $l^{\prime} \leq l$. Using the density property of $\left(\underline{n}, \bar{\gamma}^{(k-1)}(\underline{n})\right)$ we get

$$
\nu(c, c+t) \geq \delta_{1}^{(k-1)}\left(t-l^{\prime}\right) \geq \delta_{1}^{(k-1)}\left(1-u_{k} / v_{k}\right) t=\delta_{1}^{(k)} t
$$

Now, $\delta_{1}^{(k)} \searrow \delta_{1}^{(0)} \prod_{i=1}^{\infty}\left(1-u_{i} / v_{i}\right) \geq \delta_{1}^{(0)}(1-1 / \lambda) \geq \delta_{1}$ as $k \rightarrow \infty$. If we interchange $o$ - and $e$-blocks above we get

$$
N(c, c+t) \leq \delta_{2}^{(k-1)}\left(t-l^{\prime}\right)+l \leq\left(\delta_{2}^{(k-1)}+u_{k} / v_{k}\right) t=\delta_{2}^{(k)} t .
$$

In this way we can prove (1)(i), (1)(ii), (2)(i) and (2)(ii) in Definition 2.2. It remains to establish (2)(iii). Let $B=\left\langle b, b^{\prime}\right\rangle$ be an $e$-block in $\left(\underline{n}, \gamma^{(k)}(\underline{n})\right)$ and let $l\left(l^{\prime}\right)$ be the weight (length) of the $o$-blocks between $b$ and $b^{\prime}$ in $\left(\underline{n}, \gamma^{(k-1)}(\underline{n})\right)$. Then as above $l \leq u_{k}|B| / v_{k} \leq u_{k} w(B) / v_{k}$ and by the induction hypothesis $w(B)-l \leq\left(|B|-l^{\prime}\right) / \delta_{1}^{(k-1)} \leq|B| / \delta_{1}^{(k-1)}$ and hence $\left(1-u_{k} / v_{k}\right) w(B) \leq|B| / \delta_{1}^{(k-1)}$, which gives $w(B) \leq|B| / \delta_{1}^{(k)}$.

Proof of Lemma 2.8. That properties (i) and (ii) in Definition 1.1 are satisfied follows from the fact that $\Gamma_{1}(\underline{n})$ satisfies the $\left(\delta_{1}, \delta_{2}\right)$-density property. It remains to establish (iii).

Write $\Delta=\varepsilon_{N}^{-1} N^{\frac{1}{1+\xi}}$. Consider the interval $[x, x+\Delta)$ in $\left[0, t_{1}\right)$. Let $q$ be the smallest positive integer such that $\gamma^{(q)}(\underline{n}) \cap[x, x+\Delta)=\emptyset$. If $q=0$, then $[x, x+\Delta)$ is entirely contained in an $o$ - or an $e$-block in $\left(\underline{n}, \gamma^{(0)}(\underline{n})\right)$. In the first case the density in $[x, x+\Delta)$ is $\geq \delta_{1}^{(0)} \geq \delta_{1}$ and there is nothing to prove. In the second case the endpoints of the $e$-block containing $[x, x+\Delta)$ must be removed at some step $\gamma^{(p-1)} \rightarrow \gamma^{(p)}$, where $\Delta \leq u_{p}$ and $v_{p} \leq L$, which gives a contradiction for sufficiently large $N$ since

$$
u_{p} \leq\left(v_{p} /(C \lambda)^{1 /(1+\xi)} \leq C^{\prime} N^{1 /(1+\xi)}\right.
$$

Hence we can assume that $q \geq 1$ and $\gamma^{(q-1)}(\underline{n}) \cap[x, x+\Delta) \neq \emptyset$. Clearly (2.20) must hold with $p=q$, since we remove endpoints in the step $\gamma^{(q-1)} \rightarrow \gamma^{(q)}$. Consider now $o$ - and $e$-blocks in $\left(\underline{n}, \gamma^{(q-1)}(\underline{n})\right)$. Let $z$ be the length of $e$-blocks wholly or partially contained in $[x, x+\Delta)$. If $z<u_{q}$ we get using (2.20) $(p=q)$

$$
\begin{aligned}
N(x, x+\Delta) & \geq \Delta^{-1} \delta_{1}^{(q-1)}(\Delta-z) \geq \delta_{1}^{(q-1)}\left(1-C^{\prime} N^{1 /(1+\xi)} / \varepsilon_{N}^{-1} N^{1 /(1+\xi)}\right) \\
& \geq \delta_{1}-o(1)
\end{aligned}
$$

by the density property for $o$-blocks in $\left(\underline{n}, \gamma^{(q-1)}(\underline{n})\right)$.

Assume that $k u_{q} \leq z \leq(k+1) u_{q}$ for some $k \geq 1$. Then from the definition of the construction of $\gamma^{(q)}$ from $\gamma^{(q-1)}$, we see that $[x, x+\Delta)$ contains at least $k o$-blocks of length $\geq v_{q}$, i.e. $y=\Delta-z \geq k v_{q}$, where $y$ is the total $o$-block length in $[x, x+\Delta)$. Now, by the density property for $\left(\underline{n}, \gamma^{(q-1)}(\underline{n})\right)$,

$$
N(x, x+\Delta) \geq \delta_{1}^{(q-1)} \frac{y}{y+z} \geq \delta_{1}^{(q-1)}\left(1-\frac{z}{y}\right) .
$$


But $z / y \leq u_{q} / v_{q}+u_{g} / y$ and $y+(k+1) u_{q} \geq y+z=\Delta$ gives $y \geq \Delta(1+(k+$ 1) $\left.u_{q} / y\right)^{-1}$, and hence

$$
\frac{u_{q}}{y} \leq\left(1+\frac{(k+1) u_{q}}{k v_{q}}\right) \frac{u_{q}}{\Delta} \leq 3 \frac{c^{\prime} N^{1 /(1+\xi)}}{\varepsilon_{N}^{-1} N^{1 /(1+\xi)}}=o(1) .
$$

Thus

$$
\left.N(x, x+\Delta) \geq \delta_{1}^{(q-1)}\left(1-u_{q} / v_{q}\right)-o(1)\right) \geq \delta_{1}-o(1) .
$$

The proof in the case where $[x, x+\Delta)$ is contained in $\left(t_{2}, L\right)$ is analogous.

Proof of Claim 2.7. Given $j \geq 1$, let $A^{(1)}, B^{(1)}, \ldots, A^{(t)}, B^{(t)}$ be the blocks in $\Gamma \in \mathscr{P}_{\jmath+1}$. Suppose that $\Gamma=R_{\jmath} \Gamma^{\prime}$, where $\Gamma^{\prime} \in \mathscr{P}_{\jmath}$ and let $A_{1}, B_{1}, \ldots, A_{\tau}, B_{\tau}$ be the blocks in $\Gamma^{\prime}$. Then $k_{\jmath}=k_{\jmath}\left(\Gamma^{\prime}\right)=\tau-t$. From the definition of the rearrangement it is clear that each $A^{(k)}$ is of the form $A^{(k)}=A_{i} A_{i+1} \ldots A_{\text {j }}$ for some $i, j, 1 \leq i \leq j \leq \tau$, and similarly for the $e$-blocks $B^{(k)}$.

Let $g_{1}=0$ and define recursively

$$
g_{j}=v_{\jmath+1}+2 u_{\jmath}+2 g_{j-1}
$$

$j \geq 2$. Also set $w_{\jmath}=v_{j+2}+g_{j}, j \geq 1$.

Consider the following statements:

(b) ${ }_{\jmath}$ : Consider two $o$-(e-)blocks in $\Gamma \in \mathscr{P}_{j}$ of length $\geq v_{k+1}+g_{\jmath}$. Then the total weight of the $e$-(o-)blocks between them is $\geq u_{k}, k \geq j+1$.

(c) ${ }_{j}$ : If we have two $o$-(e-)blocks $C$ and $C^{\prime}$ in $\Gamma \in \mathscr{P}_{j+1}$, such that the weight of the $e$-(o-)blocks between them is $=x$, and if $\Gamma=R_{\jmath} \Gamma^{\prime}$, there are $o$ - $\left(e\right.$-)blocks $C_{*}$ and $C_{*}^{\prime}$ in $\Gamma^{\prime},\left|C_{*}\right| \geq|C|-\left(w_{j}+2 u_{j+1}\right),\left|C_{*}^{\prime}\right| \geq\left|C^{\prime}\right|-\left(w_{j}+u_{j+1}\right)$ such that the weight of the $e-(o-)$ blocks between $C_{*}$ and $C_{*}^{\prime}$ in $\Gamma^{\prime}$ is $<x+u_{j+1}$.

Since $v_{2}>v_{1},(\mathrm{~b})_{1}$ follows from Lemma 2.1 .

The strategy is now to prove that (b) and the definition of the rearrangement procedure imply the claim for this $j$ as well as (c) ${ }_{j}$. We then show that $(\mathrm{b})_{j+1}$ follows from (b) $)_{1}$ and (c) $)_{1}, \ldots,(\mathrm{c})_{3}$, and this complates the proof by induction.

As noted above an $o$-block $A^{(k)}$ in $\Gamma=R_{j} \Gamma^{\prime} \in \mathscr{P}_{j+1}, \Gamma^{\prime} \in \mathscr{P}_{j}$, is of the form $A^{(k)}=A_{i} \ldots A_{j}$, where $A_{i}, \ldots, A_{j}$ are $o$-blocks in $\Gamma^{\prime}$. Assume that $w\left(A_{\imath}\right)<u_{\jmath+1}$. Since $A_{\imath-1}$ and $A_{i}$ are not joined, $A_{\imath}$ cannot move to the left. Hence when we get to $A_{i}$ we do the rearrangement

$$
\ldots B_{\imath-1} A_{\imath} B_{i} A_{i+1} B_{i+1} \ldots \rightarrow \ldots B_{\imath-1} B_{\imath} A_{\imath} A_{\imath+1} B_{i+1} \ldots .
$$

If $i+1<j$, then $w\left(B_{\imath+1}\right)<u_{j+1}$, since $w\left(A_{\imath} A_{\imath+1}\right) \geq u_{\jmath}+u_{j}=u_{j+1}$. Hence the next rearrangement must be

$$
\ldots B_{i-1} B_{\imath} A_{\imath} A_{i+1} B_{\imath+1} A_{i+2} B_{\imath+2} \ldots \rightarrow \ldots B_{i-1} B_{\imath} A_{\imath} A_{\imath+1} A_{i+2} B_{i+1} B_{i+2} \ldots .
$$

It follows from (b) $)_{j}$ that both $A_{\imath+1}$ and $A_{\imath+2}$ cannot have length $\geq w_{\jmath}$. After this rearrangement no more $o$-block can be joined with $A_{2} A_{i+1} A_{i+2}$ unless $i+3=\tau$ and $w\left(A_{\tau}\right)<u_{j+1}$, in which case $A_{\tau}$ will be joined with $A_{i} A_{i+1} A_{i+2}$.

If $w\left(A_{i}\right) \geq u_{j+1}$ we apply the same argument as above with $A_{i} A_{i+1}$ replaced by $A_{i}$.

This argument shows that there are seven possibilities for $A^{(k)}$, and we list them below. In each case we specify the "assigned" block $A_{*}^{(k)}$ used in (c) . 
1) $A^{(k)}=A_{i} ; \pi_{1} \Gamma=\ldots B_{i-1} A_{i} B_{i} \ldots ; A_{*}^{(k)}=A_{i}$.

2) $A^{(k)}=A_{i} A_{i+1}, \pi_{1} \Gamma=\ldots B_{i-1} B_{\imath} A_{i} A_{i+1} B_{i+1} \ldots ; w\left(A_{i}\right)<u_{\jmath+1} ; A_{*}^{(k)}=A_{i+1}$.

3) $A^{(k)}=A_{\imath} A_{i+1} A_{i+2} ; \pi_{1} \Gamma=\ldots B_{i-1} B_{i} A_{i} A_{i+1} A_{i+2} B_{i+1} B_{i+2} \ldots$; $w\left(A_{i}\right)<u_{j+1} ; w\left(B_{i+1}\right)<u_{j+1} ; \min \left(\left|A_{i+1}\right|,\left|A_{i+2}\right|\right)<w_{j} ; A_{*}^{(k)}=A_{i+1}$ if $\left|A_{i+1}\right| \geq\left|A_{i+2}\right|$, otherwise $A_{*}^{(k)}=A_{i+2}$.

4) $A^{(k)}=A_{i} A_{i+1} A_{i+2} A_{i+3} ; \pi_{1} \Gamma=\ldots B_{i-1} B_{i} A_{\imath} A_{i+1} A_{i+2} A_{i+3} B_{i+1} B_{i+2} B_{\imath+3}$; $w\left(A_{i}\right)<u_{j+1} ; w\left(B_{i+1}\right)<u_{j+1} ; w\left(A_{i+3}\right)<u_{j+1} ; i+3=\tau$; $\min \left(\left|A_{\imath+1}\right|,\left|A_{\imath+2}\right|\right)<w_{j} ; A_{*}^{(k)}=A_{i+1}$ if $\left|A_{\imath+1}\right| \geq\left|A_{\imath+2}\right|$, otherwise $A_{*}^{(k)}=A_{i+2}$.

5) $A^{(k)}=A_{i} A_{i+1} ; \pi_{1} \Gamma=\ldots B_{\imath-1} A_{i} A_{i+1} B_{i} B_{i+1} \ldots ; w\left(A_{i}\right) \geq u_{j+1}$; $w\left(B_{i}\right)<u_{j+1} ; \min \left(\left|A_{i}\right|,\left|A_{i+1}\right|\right)<w_{j} ; A_{*}^{(k)}=A_{i}$ if $\left|A_{i}\right| \geq\left|A_{\imath+1}\right|$, otherwise $A_{*}^{(k)}=A_{\imath+1}$.

6) $A^{(k)}=A_{i} A_{i+1} A_{i+2} ; \pi_{1} \Gamma=\ldots B_{\imath-1} A_{i} A_{i+1} A_{i+2} B_{i} B_{i+1} B_{i+2} ; w\left(A_{i}\right) \geq u_{j+1}$; $w\left(B_{i}\right)<u_{j+1} ; w\left(A_{i+2}\right)<u_{j+1}, i+2=\tau ; \min \left(\left|A_{i}\right|,\left|A_{i+1}\right|\right)<w_{j} ; A_{*}^{(k)}=A_{\imath}$ if $\left|A_{i}\right| \geq\left|A_{i+1}\right|$, otherwise $A_{*}^{(k)}=A_{i+1}$.

7) $A^{(k)}=A_{i} A_{i+1} ; \pi_{1} \Gamma=\ldots B_{i-1} A_{i} A_{i+1} B_{i} B_{i+1} ; w\left(A_{i}\right) \geq u_{j+1} ; w\left(A_{\imath+1}\right)<u_{j+1}$; $i+1=\tau ; A_{*}^{(k)}=A_{i}$.

A similar argument shows that there are six possibilities for an $e$-block $B^{(k)}$ in $\Gamma \in \mathscr{P}_{j+1}, \Gamma=R_{j} \Gamma^{\prime}, \Gamma^{\prime} \in \mathscr{P}_{j}$.

1) $B^{(k)}=B_{i} ; \pi_{1} \Gamma=\ldots A_{i} B_{i} A_{i+1} \ldots ; B_{*}^{(k)}=B_{i}$.

2) $B^{(k)}=B_{\imath} B_{i+1}, \pi_{1} \Gamma=\ldots A_{i} A_{i+1} B_{i} B_{i+1} A_{i+2} \ldots ; w\left(B_{i}\right)<u_{j+1} ; B_{*}^{(k)}=B_{\imath+1}$.

3) $B^{(k)}=B_{i} B_{i+1} B_{\imath+2} ; \pi_{1} \Gamma=\ldots A_{i} A_{i+1} B_{i} B_{i+1} B_{i+2} A_{i+2} A_{i+3} \ldots$; $w\left(B_{i}\right)<u_{j+1} ; w\left(A_{i+2}\right)<u_{j+1} ; \min \left(\left|B_{i+1}\right|,\left|B_{i+2}\right|\right)<w_{j} ; B_{*}^{(k)}=B_{i+1}$ if $\left|B_{i+1}\right| \geq\left|B_{i+2}\right|$, otherwise $B_{*}^{(k)}=B_{i+2}$.

4) $B^{(k)}=B_{i} B_{i+1} ; \pi_{1} \Gamma=\ldots A_{\imath} B_{i} B_{i+1} A_{i+1} A_{i+2} \ldots ; w\left(B_{\imath}\right) \geq u_{\jmath+1}$; $w\left(A_{i+1}\right)<u_{j+1} ; \min \left(\left|B_{i}\right|,\left|B_{\imath+1}\right|\right)<w_{j} ; B_{*}^{(k)}=B_{i}$ if $\left|B_{i+1}\right| \geq\left|B_{\imath+2}\right|$, otherwise $B_{*}^{(k)}=B_{i+1}$.

5) $B^{(k)}=B_{i} B_{i+1} ; \pi_{1} \Gamma=\ldots A_{\imath} B_{i} B_{i+1} A_{i+1} ; i+1=\tau ; w\left(A_{i+1}\right)<u_{j+1}$; $\left|B_{i}\right|<w_{\jmath} ; B_{*}^{(k)}=B_{i+1}$.

6) $B^{(k)}=B_{i} B_{i+1} B_{i+2} ; \pi_{1} \Gamma=\ldots B_{i-1} A_{i} A_{i+1} A_{i+2} B_{i} B_{i+1} B_{i+2} ; i+2=\tau$; $w\left(B_{i}\right)<u_{j+1} ; w\left(A_{i+2}\right)<u_{j+1} ;\left|B_{i+2}\right|<w_{j} ; B_{*}^{(k)}=B_{i+2}$.

Checking all the cases we see that (c) ${ }_{j}$ holds. The fact that we need $<x+u_{i+1}$ in general and not $<x$ can be seen for example in case 3) for $o$-blocks when $A_{*}^{(k)}=A_{i+2}$. We will now prove $\left(b_{j+1}\right.$. Suppose that there are two $o$-(e-)blocks $C$ and $C^{\prime}$ in $\Gamma \in \mathscr{P}_{j+1}$ of length $\geq v_{k+1}+g_{j+1}$, such that the weight of the $e$-(o-)blocks between them is $<u_{k}, k \geq j+2$. We will show that this leads to a contradiction. $\Gamma=R_{j} \Gamma^{\prime}$ for some $\Gamma^{\prime} \subset \mathscr{P}_{j}$. By (c) $)_{j}$ there are $o$-(e-)blocks $C_{*}$ and $C_{*}^{\prime}$ in $\Gamma^{\prime}$ of length $\geq v_{k+1}+g_{j+1}-\left(w_{j}+2 u_{j+1}\right)$ such that the weight of the $e$-(o-)blocks between $C_{*}$ and $C_{*}^{\prime}$ is $<u_{k}+u_{j+1}$. Continuing in the same way, using $(\mathrm{c})_{j-1}, \ldots,(\mathrm{c})_{1}$, we 
find that there are two $o$ - $\left(e\right.$-)blocks $C_{\dagger}$ and $C_{\dagger}^{\prime}$ in $\Gamma_{\dagger}$, where $\Gamma=\mathscr{B}_{j} \Gamma_{\dagger}, \Gamma_{\dagger} \in \mathscr{P}_{1}$, of length $\geq v_{k+1}+g_{\jmath+1}-\sum_{i=1}^{j}\left(w_{\imath}+u_{j+1}\right)=v_{k+1}$, such that the total weight of the $e$ - $\left(o\right.$-)blocks between them is $<u_{k}+\sum_{i=1}^{j} u_{j+1} \leq u_{k}+u_{\jmath+2} \leq u_{k+1}$. Hence we have two $o$ - $(e-)$ blocks in $\Gamma_{+} \in \mathscr{P}_{1}$ of length $\geq v_{k+1}$ such that the length of the $e$-(o-)blocks between them is $<u_{k+1}$. This contradicts (b) ${ }_{1}$.

We now have all the facts we need to prove Claim 2.7. Given that the old $o$-point in a pair of old partition points lies in a certain $o$-block $A^{(k)}$ in $\Gamma$, we want to show that the number of possible positions for the pair is $\leq \exp \left(c_{1}+c_{2} j\right)$. Let $x$ and $y$ be the distance from the old $o$-point to the left and right endpoint of $A^{(k)}$ respectively. If $1 \leq x<u_{j+1}$ going through all the possible cases for $B^{(k-1)}$ and $A^{(k)}$ we see that the old $e$-point in the pair has distance $<w_{\jmath}+u_{j+1}$ to either the left or the right endpoint of $B^{(k-1)}$. This gives $\leq u_{j+1} \cdot 2\left(w_{j}+u_{j+1}\right)$ possibilities. If $u_{\jmath+1} \leq x<w_{j}+u_{j+1}$, we see by checking all cases that the distance from the old $e$-point to the right endpoint of $A^{(k)}$ is $<u_{j+1}$, giving $\leq w_{\jmath} \cdot u_{\jmath+1}$ possibilities. If $x \geq w_{j}+u_{\jmath+1}$ then we see that $y \leq w_{j}$. If $y \geq u_{j+1}$, then the distance from the old $e$-point to the right endpoint of $A^{(k)}$ is $<u_{j+1}$, and if $y<u_{j+1}$ the distance is $<w_{j}+u_{j+1}$ giving $\leq 2 w_{j} \cdot u_{j+1}$ possibilities. The total number of possibilities for the pair is thus $\leq 5 u_{j+1}\left(w_{j}+\right.$ $\left.u_{j+1}\right)$.

The definition of $g_{j}$ and $w_{j}$ give

$$
\begin{aligned}
w_{j}+u_{j+1} & =v_{j+2}+\sum_{i=2}^{j+1} 2^{j+1-\imath}\left(v_{i}+u_{i}\right) \\
& =C(\xi) \lambda\left[2^{(1+\xi)(j+1)}+2^{j} 2^{\xi}\left(2^{\xi j}-1\right) /\left(2^{\xi}-1\right)\right]+j 2^{j} \\
& =C(\xi)^{2} \lambda 2^{(1+\xi) j}\left[2^{1+\xi}-1+\left[j /\left(C(\xi)^{2}\right)-1\right] 2^{-\xi j}\right] .
\end{aligned}
$$

A simple computation shows that this is $\leq 5^{-1} 2^{(1+\xi) j} \exp \left(c_{1}\right)$. Hence the total number of possibilities is $\leq 2^{(2+\xi) \jmath} \exp \left(c_{1}\right)=\exp \left(c_{1}+c_{2} j\right)$.

Proof of Lemma 2.9. Let $A_{1}, B_{1}, \ldots, A_{s}, B_{s}$ be the blocks in $\Gamma_{1}(\underline{n})$. Assume that $s \geq 2$ so that $t_{1}<t_{2}$, otherwise there is nothing to prove. Let $q \geq 1$ be defined by $k_{j}=0$ if $j>q$ and $k_{q} \geq 1$. Checking the different cases, as described in the proof of Claim 2.7, we see that in each step $\mathscr{P}_{J} \rightarrow \mathscr{P}_{j+1}$, the infinitely long blocks (containing $A_{1}$ and $B_{s}$ respectively) cannot "grow" by more than $w_{j}+u_{j+1}$. Hence

$$
\Delta(\underline{n})=\sum_{j=1}^{s-1}\left(\left|B_{j}\right|+\left|A_{j+1}\right|\right) \leq 2 \sum_{j=1}^{q}\left(w_{j}+u_{j+1}\right) .
$$

From (2.22) we get

$$
\Delta(\underline{n}) \leq \frac{2}{5} e^{c_{1}} \sum_{j=1}^{q} 2^{(1+\xi) \jmath} \leq \frac{4}{5} e^{c_{1}} 2^{(1+\xi) q}
$$

with $c_{1}$ given by (2.10). Clearly $\sigma(\underline{k}(\underline{n})) \geq q$ and consequently

$$
\sigma(\underline{k}(\underline{n})) \geq \frac{\log \Delta(\underline{n})+\log \frac{5}{4}-c_{1}}{(1+\xi) \log 2} .
$$




\section{Proof of the Energy Estimate for the Lattice Gas}

In this section we will show how Theorem 1.4 follows from Proposition 2.10. Choose $\quad \kappa \in(0,1), \quad \lambda>2$ and $\xi>0$ and take $\varrho \in(1 / \lambda, 1-1 / \lambda)$. Let $Q_{N}^{*}=\left\{\underline{n} \in Q_{N, \varrho} ; n_{i}>1\right.$ some $\left.i\right\}$, so that $P_{N, \varrho, H^{d}}\left(Q_{N}^{*}\right)=0$. Note that for a configuration in $Q_{N, \varrho} \backslash Q_{N}^{*}$ the weight of a block is equal to its length. As our basic partition we take $\gamma^{(0)}(\underline{n})=\left\{j \in Z ; n_{j} \neq n_{j+1}\right\}$, i.e. a block in $\left(\underline{n}, \gamma^{(0)}(\underline{n})\right)$ consists of either just 1's (o-block) or just 0 's (e-block). Clearly the basic partition satisfies the $(1,0)$-density property.

We will now show that the estimate (2.14) holds for each $\underline{n} \in Q_{N, \varrho} \backslash Q_{N}^{*}$, where $d_{1}, d_{2}$ satisfy (2.15) under the conditions of Theorem 1.4.

Lemma 3.1. If $H^{d}$ is given by (1.3) and $H=H^{d}$, then (2.14) holds with

$$
\begin{aligned}
& d_{1}=2 \beta\left(J_{1}-1+\kappa_{1}\left(-1+\lambda^{-2}\right)-\kappa_{2} \lambda^{-1}-8\|J\|_{*}\right), \\
& d_{2}=2 \beta \mu^{2}(\lambda) \log 2,
\end{aligned}
$$

where $\kappa_{1}, \kappa_{2}$ are numerical constants.

Assume that the lemma is valid. Then

$$
K_{2}=d_{2}-c_{2}=2\left(\beta \mu^{2}(\lambda)-1-\xi / 2\right) \log 2,
$$

by (2.10) and (3.1) and this is positive provided $\beta \mu^{2}(\lambda)>1+\xi / 2$. We see that the second condition $K_{1}>0$ in (2.15) holds if $J_{1}>J_{1}^{*}$, where

$$
J_{1}^{*}=1+\kappa_{1}\left(1-\lambda^{-2}\right)+\kappa_{2} \lambda^{-1}+\frac{1}{2 \beta}\left[c_{1}-\log \left(\exp \left(\kappa K_{2}\right)-1\right)\right] .
$$

Theorem 1.4 now follows from Proposition 2.10 and from (3.2) and (2.17) it follows that $\psi$ is given by (1.6); furthermore $D=2^{1+\xi} \exp \left(c_{1}\right)$.

To prove Corollary 1.5 we use $\exp x-1 \geq x$ in (3.3) and choose $\xi / 2=$ $\beta(1-4 / \lambda)-1$. Then $\beta \mu^{2}(\lambda)-1-\xi / 2>0$. Put $\lambda=5 \beta(\beta-1)^{-1}$ so that $\xi=2(\beta-1) / 5$ and $\kappa=1 / 2$. A simple computation shows that $C(\xi) \leq(\xi \log 2)^{-1}(1+\xi \log 2)$ and we obtain

$$
J_{1}^{*} \leq C_{1}+\frac{5}{2} \beta^{-1} \log \frac{1}{\beta-1},
$$

where $C_{1}$ is a numerical constant. Now, if

$$
\beta-1>\exp \left(2 C_{1} / 5\right) \exp \left(-2 J_{1} / 5\right)
$$

and $J_{1}>C_{1}$, then (3.4) and (3.5) imply $J_{1}>J_{1}^{*}$, and hence the conclusion of the theorem, which in turn gives $M\left(\beta, J_{1}\right)>0$. Thus the estimate (1.8) in Corollary 1.5 must hold.

To prove Lemma 3.1 we need to introduce some more notation. For $\underline{n} \in Q_{N, \varrho}$, let $r_{0}(\underline{n})=0$ and

$$
r_{j}(\underline{n})=\sum_{i=1}^{j} k_{i}(\underline{n}), \quad 1 \leq j \leq p,
$$

i.e. $r_{j}(\underline{n})$ is the number of elementary rearrangements performed in going from $\Gamma_{1}(\underline{n})$ to $\Gamma_{j+1}^{j}(\underline{n})$. The total number of rearrangements is $s(\underline{k}(\underline{n}))=r_{p}(\underline{n})$. If $r_{j-1}<i \leq r_{j}$ 
we put

$$
\Gamma^{(i)}(\underline{n})=S_{\jmath}^{\imath-r_{\jmath-1}} \Gamma_{j}(\underline{n})
$$

so that $\Gamma^{(i)}(\underline{n})$ is the configuration obtained after $i$ rearrangements starting from $\Gamma_{1}(\underline{n})$.

Lemma 3.1 will follow if we can prove that

$$
\Delta H=H\left(\pi_{1} \Gamma^{(i-1)}(\underline{n})\right)-H\left(\pi_{1} \Gamma^{(\imath)}(\underline{n})\right) \geq d_{1}+d_{2} j
$$

when $r_{j-1}<i \leq r_{j}$.

Let $\phi$ be a pair potential and $C=\left(e_{1}, \ldots, e_{x}\right), C^{\prime}=\left(f_{1}, \ldots, f_{x^{\prime}}\right)$ two blocks with $C$ to the left of $C^{\prime}$. The interaction energy between $C$ and $C^{\prime}$, when the distance between the blocks is $z$, is then

$$
E_{\phi}\left(C, C^{\prime}, z\right)=\sum_{i=0}^{x-1} \sum_{j=1}^{x^{\prime}} e_{x-i} f_{\jmath} \phi(i+j+z) .
$$

The $i^{\text {th }}$ rearrangement is either of the form $C_{0} A_{1} B_{1} A_{2} B_{2} A_{3} C_{1} \rightarrow C_{0} A_{1} A_{2} B_{1} B_{2} A_{3} C_{1}$ or $C_{1} A_{3} B_{2} A_{2} B_{1} A_{1} C_{0} \rightarrow C_{1} A_{3} B_{2} B_{1} A_{2} A_{1} C_{0}$, where $A_{i}, i=1,2,3$ are $o$-blocks, $B_{i}, i=1,2$ are $e$-blocks, and $C_{0}, C_{1}$ contains all other blocks. Let $x_{i}=\left|A_{i}\right|$ and $y_{i}=\left|B_{\imath}\right|$. We than have

$$
\begin{gathered}
x_{i}, y_{i} \geq u_{j}, \\
\max \left(x_{2}, y_{1}\right)<u_{\jmath+1} .
\end{gathered}
$$

Now, write $J^{1}(r)=J_{1}-1$ if $r=1, J^{1}(r)=0$, if $r \geq 2, J^{2}(r)=1 / r^{2}, r \geq 1$, and $J^{3}(r)=0$ if $r=1, J^{3}(r)=J(r)-1 / r^{2}$ if $r \geq 2$, so that $J=J^{1}+J^{2}+J^{3}$. Put

$$
\begin{aligned}
\Delta H^{k}= & E_{J^{k}}\left(C_{0} A_{1}, B_{1}, 0\right)-E_{J^{k}}\left(C_{0} A_{1} A_{2}, B_{1}, 0\right) \\
& +E_{J^{k}}\left(B_{1}, A_{2} B_{2} A_{3} C_{1}, 0\right)-E_{J^{k}}\left(B_{1}, B_{2} A_{3} C_{1}, 0\right) \\
& +E_{J^{k}}\left(C_{0} A_{1}, A_{2}, y_{1}\right)-E_{J^{k}}\left(C_{0} A_{1}, A_{2}, 0\right) \\
& +E_{J^{k}}\left(A_{2} B_{2} A_{3} C_{1}, 0\right)-E_{J^{k}}\left(A_{2}, B_{2} A_{3} C_{1}, y_{1}\right) .
\end{aligned}
$$

Then $\Delta H=\Delta H^{1}+\Delta H^{2}+\Delta H^{3}$. We get immediately that

$$
\begin{aligned}
& \Delta H^{1}=2 \beta\left(J_{1}-1\right), \\
& \Delta H^{3} \geq-16 \beta\|J\|_{*},
\end{aligned}
$$

where we have used $\sum_{r=2}^{\infty} r\left|J^{3}(r)\right|=\|J\|_{*}$ in (3.9).

Let $A_{\imath}=\left(n_{a_{i}}, \ldots, n_{b_{\imath}-1}\right), i=1,2,3$ and $B_{\imath}=\left(n_{b_{i}}, \ldots, n_{a_{\imath+1}-1}\right), i=1,2$, and write $n_{1, k}=1-n_{b_{1}-k}, n_{2, k}=1-n_{a_{2}+k-1}, n_{3, k}=1-n_{b_{2}-k}, n_{4, k}=1-n_{a_{3}+k-1}$, $n_{5, k}=n_{b_{1}+k-1}, n_{6, k}=n_{a_{2}-k}$ and $n_{7, k}=n_{b_{2}+k-1}$. Then the density property gives

$$
0 \leq \sum_{i=1}^{k} n_{r, i} \leq \delta_{2} k
$$

$\left(\delta_{1}=1 / \lambda\right), r=1, \ldots, 7$ for $1 \leq k \leq x_{1}, 1 \leq k \leq x_{2}, 1 \leq k \leq x_{2}, 1 \leq k \leq x_{3}$, $1 \leq k \leq y_{1}, 1 \leq k \leq y_{1}$ and $1 \leq k \leq y_{2}$ respectively. 
Using this notation we have

(i) $E_{J^{2}}\left(C_{0} A_{1}, B_{1}, 0\right)-E_{J^{2}}\left(C_{0} A_{1} A_{2}, B_{1}, 0\right)$

$$
\begin{aligned}
= & -\sum_{i=1}^{\infty} \sum_{j=1}^{y_{1}} \frac{\left(1-n_{1, i}\right) n_{5, j}}{(i+j-1)^{2}}+\sum_{i=1}^{x_{2}} \sum_{j=1}^{y_{1}} \frac{\left(1-n_{3, i}\right) n_{5, j}}{(i+j-1)^{2}} \\
& +\sum_{i=1}^{\infty} \sum_{j=1}^{y_{1}} \frac{\left(1-n_{1, i}\right) n_{5, j}}{\left(i+j+x_{2}-1\right)^{2}} \\
\geq & \sum_{i=1}^{x_{2}} \sum_{j=1}^{y_{1}} \frac{n_{3, i} n_{5, j}}{(i+j-1)^{2}}-\sum_{i=x_{2}+1}^{\infty} \sum_{j=1}^{y_{1}} n_{5, j}\left(\frac{1}{(i+j-1)^{2}}-\frac{1}{\left(i+j+x_{2}-1\right)^{2}}\right) \\
\doteq & -\Sigma_{1}-\Sigma_{2} ;
\end{aligned}
$$

(ii) $E_{J^{2}}\left(B_{1}, A_{2} B_{2} A_{3} C_{1}, 0\right)-E_{J^{2}}\left(B_{1}, B_{2} A_{3} C_{1}, 0\right)$

$$
\geq-\sum_{i=1}^{y_{1}} \sum_{j=1}^{x_{2}} \frac{\left(1-n_{2,2}\right) n_{6, j}}{\left(i+j+x_{2}-1\right)^{2}} \doteq-\Sigma_{3} \text {; }
$$

(iii) $E_{J^{2}}\left(C_{0} A_{1}, A_{2}, y_{1}\right)-E_{J^{2}}\left(C_{0} A_{1}, A_{2}, 0\right)$

$$
=\sum_{i=1}^{\infty} \sum_{j=1}^{x_{2}}\left(1-n_{1, j}\right)\left(1-n_{2, j}\right)\left(\frac{1}{(i+j-1)^{2}}-\frac{1}{\left(i+j+y_{1}-1\right)^{2}}\right) \doteq \Sigma_{4}
$$

(iv) $E_{J^{2}}\left(A_{2}, B_{2} A_{3} C_{1}, 0\right)-E_{J^{2}}\left(A_{2}, B_{2} A_{3} C_{1}, y_{1}\right)$

$$
\begin{aligned}
\geq & \sum_{i=1}^{x_{2}} \sum_{j=1}^{y_{2}} n_{7, \jmath}\left(\frac{1}{(i+j-1)^{2}}-\frac{1}{\left(i+j+y_{1}-1\right)^{2}}\right) \\
& -\sum_{i=1}^{x_{2}} \sum_{j=1}^{\infty}\left(\frac{1}{\left(i+j+y_{2}-1\right)^{2}}-\frac{1}{\left(i+j+y_{1}+y_{2}-1\right)^{2}}\right) \\
\doteq & -\Sigma_{5}-\Sigma_{6} .
\end{aligned}
$$

Now,

$$
\begin{aligned}
-\Sigma_{3}+\Sigma_{4} & \geq \sum_{i=1}^{y_{1}} \sum_{j=1}^{x_{2}} \frac{\left(1-n_{1, \imath}-n_{6, \imath}\right)\left(1-n_{2, j}\right)}{(i+j-1)^{2}}-\sum_{i=1}^{y_{1}} \sum_{j=1}^{x_{2}} \frac{1}{\left(i+j+y_{1}-1\right)^{2}} \\
& \doteq \Sigma_{7}-\Sigma_{8} .
\end{aligned}
$$

Hence

$$
\Delta H^{2} \geq \Sigma_{7}-\Sigma_{1}-\Sigma_{2}-\Sigma_{5}-\Sigma_{6}-\Sigma_{8}
$$

Let $f$ be a non-negative, decreasing and convex function and consider

$$
S=\sum_{i=a}^{b} \sum_{j=c}^{d} \alpha_{i} \beta_{j} f(i+j) .
$$

Put $A_{k}=\sum_{i=a}^{k} \alpha_{i}, B_{k}=\sum_{j=c}^{k} \beta_{j}$. Assume that $0 \leq \tilde{A}_{\imath} \leq A_{\imath} \leq A_{i}^{*}, i=a, \ldots, b$, $\tilde{A}_{a-1}=A_{a-1}^{*}=0, \tilde{B}_{i} \leq B_{\imath} \leq B_{i}^{*}, i=c, \ldots, d, \tilde{B}_{c-1}=B_{c-1}^{*}=0$, and write 
$\tilde{\alpha}_{\imath}=\tilde{A}_{\imath}-\tilde{A}_{i-1}, \tilde{\beta}_{i}=\tilde{B}_{i}-\tilde{B}_{i-1}, \alpha_{i}^{*}=A_{i}^{*}-A_{i-1}^{*}, \beta_{i}^{*}=B_{i}^{*}-B_{i-1}^{*}$. Summation by parts then gives

$$
\sum_{i=a}^{b} \sum_{j=c}^{d} \tilde{\alpha}_{i} \tilde{\beta}_{j} f(i+j) \leq S \leq \sum_{i=a}^{b} \sum_{j=c}^{d} \alpha_{i}^{*} \beta_{j}^{*} f(i+j) .
$$

Using (3.10) and (3.12) we obtain

$$
\begin{aligned}
& \Sigma_{1} \leq \delta_{2}^{2} \sum_{i=1}^{x_{2}} \sum_{j=1}^{y_{1}-1} \frac{1}{(i+j)^{2}} \\
& \Sigma_{2} \leq \delta_{2} \sum_{i=x_{2}+1}^{2 x_{2}} \sum_{j=1}^{y_{1}-1} \frac{1}{(i+j)^{2}}, \\
& \Sigma_{5} \leq \delta_{2} \sum_{i=1}^{x_{2}} \sum_{j=1}^{y_{1}} \frac{1}{(i+j)^{2}}, \\
& \Sigma_{7} \leq\left(1-2 \delta_{2}\right)\left(1-\delta_{2}\right) \sum_{i=1}^{y_{1}} \sum_{j=1}^{x_{2}-1} \frac{1}{(i+j)^{2}}+\left(1-2 \delta_{2}\right) \sum_{i=1}^{y_{1}} \frac{1}{i^{2}} \\
& \Sigma_{6}=\sum_{i=1}^{x_{2}} \sum_{j=y_{2}}^{y_{1}+y_{2}-1} \frac{1}{(i+j)^{2}}, \\
& \Sigma_{8}=\sum_{i=y_{1}}^{2 y_{1}-1} \sum_{j=1}^{x_{2}} \frac{1}{(i+j)^{2}} .
\end{aligned}
$$

To estimate these sums we use the following result which follows from Eulers summation formula. If $a+c \geq 2$, then

$$
\begin{aligned}
\sum_{i=1}^{b} \sum_{j=c}^{d} \frac{1}{(i+j)^{2}}= & \log \frac{(a+d)(b+c)}{(a+c)(b+d)}+\frac{1}{a+c}-\frac{1}{b+d} \\
& +\frac{5}{12}\left(\frac{1}{(a+c)^{2}}+\frac{1}{(b+d)^{2}}\right) \\
& +\frac{1}{12}\left(\frac{1}{(a+d)^{2}}+\frac{1}{(b+c)^{2}}\right)+\frac{1}{6}\left(\frac{1}{(a+c)^{3}}+\frac{1}{(b+d)^{3}}\right) \\
& +\frac{1}{24}\left(\frac{1}{(a+c)^{4}}+\frac{1}{(b+c)^{4}}-\frac{1}{(a+d)^{4}}-\frac{1}{(b+c)^{4}}\right)+R
\end{aligned}
$$

where $-1 / 100 \leq R \leq 0$.

Using (3.7) and (3.14) to estimate the sums in (3.13), (3.11) gives

$$
\Delta H^{2} \geq 2 \beta\left[1-4 \delta_{2}+\delta_{2}^{2}\right]\left[j \log 2-C_{1}\right]-2 \beta C_{2} \delta_{2},
$$

where $C_{1}$ and $C_{2}$ are numerical constants. $\left(C_{1} \approx 2\right.$ and $C_{2} \approx 6$ will do, but better values can be obtained with more numerical work.) Combining (3.8), (3.9) and (3.15) we obtain (3.6), with $\kappa_{1}=C_{1}$ and $\kappa_{2}=4 C_{1}-C_{2}$. 


\section{Proof of the Energy Estimate for the Continuous Gas}

To prove Theorem 1.2 we want to apply Proposition 2.10 to the lattice gas model with Hamiltonian $H_{\phi}^{c}(\underline{n}, \beta)$ given by (1.2) where $\phi$ is given by (1.1).

Given $\underline{n} \in Q_{N, \varrho}$ we define the basic partition $\gamma^{(0)}(\underline{n})$ as follows. If $n_{i-1} \geq 1$, $n_{i}=n_{i+1}=0$, then $i+1 \in \gamma^{(0)}(\underline{n})$; if $n_{i-1}=n_{i}=0$ and $n_{\imath+1} \geq 1$, then $i+1 \in \Gamma^{(0)}(\underline{n})$. It follows immediately that $\left(\underline{n}, \gamma^{(0)}(\underline{n})\right)$ satisfied the $(1 / 2,0)$-density property.

The key energy estimate is the content of

Lemma 4.1. If $0<\varrho<1 / 4$ and $\lambda \geq 7$, then

(i) there is a $Q_{N}^{*} \subseteq Q_{N, \varrho}$ such that

$$
P_{N, \varrho, \phi}^{c}\left\{\underline{x}, \underline{n}(\underline{x}) \in Q_{N}^{*}\right\} \leq 2 \varrho^{-1} N^{2-c_{1}^{*} \beta},
$$

where $c_{1}^{*}$ only depends on the parameters in $\phi$, and

(ii) there is a diffeomorphism $\mathscr{R}^{*}: \Omega(\underline{n}) \rightarrow \Omega(\mathscr{R} \underline{n})$ with Jacobian $=1$ such that

$$
H_{\phi}(\underline{x})-H_{\phi}\left(\mathscr{R}^{*}(\underline{x})\right) \geq \gamma_{1} s(\underline{k})+\gamma_{2} \sigma(\underline{k}),
$$

if $\underline{n}(\underline{x}) \in\left(Q_{N, \varrho} \backslash Q_{N}^{*}\right) \cap\left\{\underline{n} ; \sigma(\underline{k}(\underline{n})) \geq c_{2}^{*} \lambda^{-1} \log N\right\}$, where $\underline{k}=\underline{k}(\underline{n}(\underline{x}))$ and $\gamma_{1}, \gamma_{2}, c_{2}^{*}$ are positive constants, which only depend on the parameters in $\phi$

Assuming the validity of this lemma we can prove Theorem 1.5. Note that

$$
H_{\phi}^{c}(\underline{n}, \beta)-H_{\phi}^{c}(\mathscr{R}(\underline{n}), \beta)=\log \frac{\int_{\Omega(\mathscr{B}(\underline{n}))} \exp \left(-\beta H_{\phi}(\underline{x})\right) d^{N} x}{\int_{\Omega(\underline{n})} \exp \left(-\beta H_{\phi}(\underline{x})\right) d^{N} x} .
$$

The integral in the numerator is greater than or equal to

$$
\int_{\mathscr{R}^{*}(\Omega(\underline{n}))} \exp \left(-\beta H_{\phi}(\underline{y})\right) d^{N} y=\int_{\Omega(\underline{n})} \exp \left(-\beta H_{\phi}\left(\mathscr{R}^{*}(\underline{x})\right)\right) d^{N} x .
$$

Here we have made the changed of variables $y=\mathscr{R}^{*}(\underline{x})$. The right-hand side of (4.3) can be written

$$
\begin{aligned}
& \int_{\Omega(\underline{n})} \exp \left(-\beta H_{\phi}(\underline{x})\right) \exp \left(\beta\left(H_{\phi}(\underline{x})-H_{\phi}\left(\mathscr{B}^{*}(\underline{x})\right)\right) d^{N} x\right. \\
& \quad \geq \exp \left(\gamma_{1} s(\underline{k})+\gamma_{2}(\underline{k})\right) \int_{\Omega(\underline{n})} \exp \left(-\beta H_{\phi}\left(\mathscr{R}^{*}(\underline{x})\right)\right) d^{N} x,
\end{aligned}
$$

where the inequality follows from (4.1). Inserting this in (4.2) gives (2.14) with $d_{1}=\beta \gamma_{1}, d_{2}=\beta \gamma_{2}$. We see that the conditions (2.15) will be satisfied provided $\beta$ is sufficiently large. The constants involved are chosen as follows: $\kappa=1 / 2$, $1+\xi=\eta_{2} \log \beta, \lambda=\exp \left(\eta_{1} \beta\right)$, where $\eta_{2}=\min \left(\gamma_{2} / 2 \log 32, \gamma_{1} / 2 \log 2\right)$ and $\eta_{1}=\gamma_{1}-\eta_{2} \log 2$. If we assume that

$$
\beta \geq \beta^{*}=\max \left(\frac{10 \log 162}{9 \gamma_{2}}, \frac{2 \log 7}{\gamma_{1}}, \frac{4}{c_{1}^{*}}, \frac{\log 2 c_{2}^{*}}{\eta_{1}}\right),
$$


then some computation shows that the conditions in Proposion 2.10 are satisfied with $\psi \geq 2$ and $D=N^{\eta_{3} \exp \left(-\eta_{1} \beta\right)}$, where $\eta_{3}=2 c_{2}^{*}$ provided $N$ is sufficiently large. Theorem 1.2 now follows from Proposition 2.10 and the estimate Lemma 4.1(i).

Proof of Lemma 4.1. The first step is the definition of $\mathscr{B}^{*}$. Consider a fixed $\underline{x} \in \Omega(\underline{n})$, $\underline{n} \in Q_{N, \varrho}$. Let $A_{1}, B_{1}, \ldots, A_{s}, B_{s}$ be the blocks in $\Gamma_{1}(\underline{n})$. If $A_{j}=\left\langle a_{j}, b_{j}\right\rangle$ and $a_{\jmath} \leq x_{k}<\ldots<x_{l}<b_{j}$, we define

$$
I\left(A_{j}\right)=\left\{x_{\imath}-\max \left(0, a_{i}\right) ; k \leq i<l\right\},
$$

i.e. $I\left(A_{j}\right)$ gives the positions of the particles in $A_{j}$ relative to the left endpoint (or the origin). $I\left(B_{\jmath}\right)$ is defined analogously if there are particles in $B_{\jmath}$, otherwise $I\left(B_{j}\right)=\emptyset$.

Fix $k, 1<k \leq s$, and let $A^{(1)}, B^{(1)}, \ldots, A^{(\tau)}, B^{(\tau)}$ be the blocks in $\Gamma^{(k)}(\underline{n})$. Then each $A^{(j)}$ has been built up from $o$-blocks in $\Gamma_{1}(\underline{n})$, i.e. $A^{(j)}=A_{t_{j}} \ldots A_{t_{j+1}-1}$. We set

$$
I\left(A^{(j)}\right)=\bigcup_{\imath=t_{j}}^{t_{\jmath+1}-1}\left(I\left(A_{i}\right)+\left|A_{t_{\jmath}} \ldots A_{i}\right|\right)
$$

which then gives the positions of the particles in $A^{(j)} . I\left(B^{(\jmath)}\right)$ is defined analogously. Recall that $\mathscr{B}(\underline{n})=A_{1} \ldots A_{s} B_{1} \ldots B_{s}$. Thus if $I\left(A_{1} \ldots A_{s}\right)=\left\{y_{\imath}\right\}_{i=-\infty}^{K}$ and $I\left(B_{1} \ldots B_{s}\right)=\left\{y_{i}-b_{1}-\sum_{j=2}^{s}\left(b_{j}-a_{j}\right)\right\}_{i=K+1}^{N}$, where $y_{i-1}<y_{i}, i \leq N$, we see that it is natural to define $\mathscr{R}^{*}(\underline{x})=\underline{y}$. It is clear that $\mathscr{R}^{*}$ is a diffeomorphism on $\Omega(\underline{n})$ with Jacobian $=1$.

Each $A_{i}$ lies in some block $A^{\left(l_{\imath}\right)}$ in $\Gamma^{(k)}(\underline{n})$, so we can talk about the distance $d_{\imath, j}^{(k)}$ between $A_{\imath}$ and $A_{j}$ in $\Gamma^{(k)}(\underline{n})$. Note that for all $i, j$,

$$
d_{\imath, j}^{(s-1)}<d^{(0)}(i, j) .
$$

The interaction energy between two blocks $C$ and $C^{\prime}$ at distance $z$ is given by

$$
E_{\phi}\left(C, C^{\prime}, z\right)=\sum_{s \in I(C), t \in I\left(C^{\prime}\right)} \phi(|C|-s+t+z),
$$

and the interaction energy between the particles within a block $C$ is

$$
E_{\phi}\left(C, C^{\prime}, 0\right)=\sum_{s, t \in I(C), s<t} \phi(t-s)
$$

For $0 \leq k<s$ we set

$$
H_{\phi, 1}^{(k)}=\sum_{1 \leq i<j \leq s} E_{\phi}\left(A_{i}, A_{j}, d_{i, j}^{(k)}\right)
$$

We consider a fixed $\underline{x} \in \Omega(\underline{n})$, and we will not indicate the dependence on $\underline{x} . H_{\phi, 1}^{(k)}$ gives the interaction energy between the particles in different original $o$-blocks after $k$ elementary rearrangements. Also, set

$$
H_{\phi, 2}^{(k)}=\sum_{i=1}^{s}\left(E_{\phi}\left(A_{\imath}, A_{\imath}, 0\right)+E_{\phi}\left(B_{\imath}, B_{\imath}, 0\right)\right),
$$


for $0 \leq k<s$, and finally

$$
\begin{aligned}
H_{\phi, 3}^{(0)} & =H_{\phi}(\underline{x})-H_{\phi, 1}^{(0)}-H_{\phi, 2}^{(0)}, \\
H_{\phi, 3}^{(s-1)} & =H_{\phi}\left(\mathscr{R}^{*}(\underline{x})\right)-H_{\phi, 1}^{(s-1)}-H_{\phi, 2}^{(s-1)} .
\end{aligned}
$$

Here $H_{\phi, 2}^{(k)}$ represents the interaction energy between particles within the same original $o$ - or $e$-blocks, and clearly it does not change during the rearrangement procedure. $H_{\phi, 3}^{(k)}, k=0, s-1$ represents the interaction energy between $e$-blocks, and between $e$ - and $o$-blocks.

We will now state some estimates which together imply (4.1). There is a $Q_{N}^{*} \subseteq$ $Q_{N, \varrho}$ satisfying Lemma 4.1(i) such that if $\underline{n}(\underline{x}) \in Q_{N, \varrho} \backslash Q_{N}^{*}, \varrho<1 / 4, \lambda \geq 7$ and $s=s(\underline{k}) \geq 2, \underline{k}=\underline{k}(\underline{n})$, then

$$
\begin{gathered}
\Delta H_{\phi, 1} \doteq H_{\phi, 1}^{(0)}-H_{\phi, 1}^{(s-1)} \geq \kappa_{1} s(\underline{k}), \\
\Delta H_{\phi, 1} \geq-\kappa_{2} s(\underline{k})+\kappa_{3} \sigma(\underline{k}), \\
\Delta H_{\phi, 3} \doteq H_{\phi, 3}^{(0)}-H_{\phi, 3}^{(s-1)} \geq-\frac{1}{2} \Delta H_{\phi, 1}-\lambda^{-1} \kappa_{4} \log N,
\end{gathered}
$$

where $\kappa_{i}, i=1, \ldots, 4$ are positive constants, which only depend on the parameters in $\phi$.

Let us first show how (4.1) follows from (4.10) to (4.12). If the right-hand side of (4.11) is $\geq \kappa_{3} \sigma(\underline{k}) / 2,(4.10)$ and (4.11) give

$$
\Delta H_{\phi, 1} \geq \frac{\kappa_{1}}{2} s(\underline{k})+\frac{\kappa_{3}}{4} \sigma(\underline{k}) .
$$

If the right-hand side of (4.11) is $<\kappa_{3} \sigma(\underline{k}) / 2$, then $s(\underline{k}) \geq \kappa_{3}\left(2 \kappa_{2}\right)^{-1} \sigma(\underline{k})$ and (4.10) gives

$$
\Delta H_{\phi, 1} \geq \frac{\kappa_{1}}{2} s(\underline{k})+\frac{\kappa_{3}}{4 \kappa_{2}} \sigma(\underline{k}) \text {. }
$$

Let $\gamma_{2}=\min \left(\kappa_{3} / 8, \kappa_{3} / 8 \kappa_{2}\right)$. Since $H_{\phi, 2}^{(0)}-H_{\phi, 2}^{(s-1)}=0$, (4.12)-(4.14) yield

$$
H_{\phi}(\underline{x})-H_{\phi}\left(\mathscr{R}^{*} \underline{x}\right) \geq \gamma_{1} s(\underline{k})+\gamma_{2} \sigma(\underline{k})+\left(\gamma_{2} \sigma(\underline{k})-\lambda^{-1} \kappa_{4} \log N\right),
$$

where $\gamma_{1}=\kappa_{1} / 4$. This gives (4.1) if we put $c_{2}^{*}=\kappa_{4} / \gamma_{2}$.

Proof of (4.10). We have

$$
\begin{aligned}
H_{\phi, 1}^{(0)}-H_{\phi, 1}^{(s-1)} & =\sum_{1 \leq i<j \leq s}\left(E_{\phi}\left(A_{i}, A_{j}, d_{i, j}^{(0)}\right)-E_{\phi}\left(A_{\imath}, A_{\jmath}, d_{i, j}^{(s-1)}\right)\right) \\
& \geq \sum_{j=1}^{s-1}\left(E_{\phi}\left(A_{j}, A_{j+1}, 1\right)-E_{\phi}\left(A_{j}, A_{j+1}, 0\right)\right) \\
& \geq 2 \kappa_{1}(s-1) \geq \kappa_{1} s .
\end{aligned}
$$

where we have used $d_{\jmath, \jmath+1}^{(s-1)}=0,(4.5)$, and the fact that $\phi(r)$ is strictly increasing when $r \geq 1$. It is clear that $\kappa_{1}$ only depends on $\phi$.

Proof of (4.11). We write $\phi(r)=\psi(r)+\xi(r)$ as in (1.1), where $\xi(r)=-A / r^{2}$. Hence $\Delta H_{\phi, 1}=\Delta H_{\psi, 1}+\Delta H_{\xi, 1}$. Inequality (4.5), (4.6) and the fact that $\psi(r)$ is increasing for $r \geq 1$, yield $\Delta H_{\psi, 1} \stackrel{\geq}{\geq} 0$ and thus

$$
\Delta H_{\phi, 1} \geq \Delta H_{\xi, 1} .
$$


For any $\underline{n} \in Q_{N, \varrho}$ define $\nu_{i}=\min \left(1, n_{i}\right)$, so that $\nu(x, y)$, defined in (2.4), is given by $\sum_{i=x}^{y-1} \nu_{i}$. If $\nu_{i}=1$, then there are particles at $x_{l}, \ldots, x_{l+n_{i}-1}$ in $[i-1, i)$, and we let $M_{i}=\left\{x_{l}\right\}$. If $\nu_{i}=0$ we let $M_{i}=\emptyset$. For each $o$-block $A_{\imath}$ in $\Gamma_{1}(\underline{n})$ we define

$$
I^{*}\left(A_{j}\right)=\bigcup_{j=a_{j}}^{b_{\jmath}-1}\left(M_{i}-\min \left(0, a_{\jmath}\right)\right), \quad j=1, \ldots, s .
$$

Then $I^{*}\left(A_{j}\right) \subset I\left(A_{j}\right)$, and $I^{*}\left(A_{j}\right)$ describes the configuration we get if we just keep one particle in every occupied box. If $A^{(j)}$ is an $o$-block in $\Gamma^{(k)}$, we define $I^{*}\left(A^{(k)}\right)$ in analogy with (4.4). Also,

$$
E_{\xi}^{*}\left(A, A^{\prime}, z\right)=\sum_{s \in I^{*}(A), t \in I^{*}\left(A^{\prime}\right)} \xi(|A|-s+t+z) .
$$

Furthermore, let

$$
H_{\xi, 1}^{*(k)}=\sum_{1 \leq i<j \leq s} E_{\xi}^{*}\left(A_{i}, A_{j}, d_{\imath, j}^{(k)}\right)
$$

Now, it is not difficult to see that

$$
\Delta H_{\xi, 1} \geq \Delta H_{\xi, 1}^{*} \doteq H_{\xi, 1}^{*(0)}-H_{\xi, 1}^{*(s-1)}
$$

To prove this, observe it suffices to prove the nonnegativity of

$$
\begin{aligned}
& E_{\xi}\left(A_{i}, A_{\jmath}, d_{i, j}^{(0)}\right)-E_{\xi}^{*}\left(A_{i}, A_{j}, d_{i, j}^{(0)}\right) \\
& \quad-\left(E_{\xi}\left(A_{i}, A_{j}, d_{i, j}^{(s-1)}\right)-E_{j}^{*}\left(A_{i}, A_{j}, d_{i, j}^{(s-1)}\right)\right)
\end{aligned}
$$

which equals

$$
\left(\sum_{\substack{t \in I\left(A_{2}\right) \backslash I^{*}\left(A_{\imath}\right) \\ s \in I\left(A_{\jmath}\right)}}+\sum_{\substack{t \in I\left(A_{i}\right) \\ s \in I\left(A_{\jmath}\right) \backslash I^{*}\left(A_{j}\right)}}\right)^{\left[\xi\left(\left|A_{i}\right|-t+s+d_{i, j}^{(0)}\right)-\xi\left(\left|A_{\imath}\right|-t+s+d_{i, j}^{(s-1)}\right)\right] .}
$$

That each term in these last sums is nonnegative follows from (4.5) and the fact that $\xi$ is increasing.

Considering the right-hand side of (4.10) means that we are trying to estimate the change in energy by just considering one particle in each box and neglecting the others. This suffices unless there are very many particles in an $o$-block compared to its length. To tackle this problem we have to consider two different cases.

Suppose that in rearrangement number $k$ from $\Gamma^{(k-1)}(\underline{n})$ to $\Gamma^{(k)}(\underline{n})$, the o-blocks $A$ and $A^{\prime}$ are joined. Define

$$
\eta_{k}=\left\{\begin{array}{l}
1 \text { if } w(A) \leq 2|A|^{5} \text { and } w\left(A^{\prime}\right) \leq 2\left|A^{\prime}\right|^{5} \\
0 \text { otherwise }
\end{array}\right.
$$

Assume first that $\eta_{k}=1$. We want to estimate the right-hand side of (4.16). Suppose that $A^{\prime}$ is moved to the left when it is joined with $A$; the other case is analogous. If $A^{\prime}=A_{g} \ldots A_{l-1}$ we let $A^{\prime \prime}=A_{l} \ldots A_{s}$, i.e. the join of all $o$-blocks 
to the right of $A_{l}$. Since $\xi$ is increasing and concave, we see that $E_{\xi}^{*}\left(A, A^{\prime}, z\right)$ and $E_{\xi}^{*}\left(A, A^{\prime}, z\right)-E_{\xi}^{*}\left(A, A^{\prime}, z+y\right)$ are increasing functions of $z \geq 1, y \geq 0$ arbitrary. Hence

$$
\begin{aligned}
H_{\xi, 1}^{*(k)}-H_{\xi, 1}^{*(k+1)} \geq & E_{\xi}^{*}\left(A, A^{\prime}, y_{1}\right)-E_{\xi}^{*}\left(A, A^{\prime}, 0\right) \\
& +E_{\xi}^{*}\left(A^{\prime}, A^{\prime \prime}, y_{2}\right)-E_{\xi}^{*}\left(A^{\prime}, A^{\prime \prime}, y_{1}+y_{2}\right),
\end{aligned}
$$

where $y_{1}$ is the length of the $e$-block, $B$, between $A$ and $A^{\prime}$, and $y_{2}$ is the length of the $e$-block $B^{\prime}$, between $A^{\prime}$ and $A_{l} \ldots$.

Suppose that $r_{j-1} \leq k<r_{j}, j \geq 1$. It follows from the density property Definition 2.2(2)(iii) that $|B| \geq \delta_{1} w(B),\left|B^{\prime}\right| \geq \delta_{1} w\left(B^{\prime}\right)$, and since $w(b)$ and $w\left(B^{\prime}\right)$ are $\geq u_{\jmath}$ we have

$$
y_{i} \geq \delta_{1} u_{\jmath}, \quad i=1,2 .
$$

Also, from the assumption $\eta_{k} \geq 1$,

$$
x_{\imath} \geq u_{j-1}^{1 / 5}, \quad i=1,2,
$$

where $x_{1}=|A|, x_{2}=\left|A^{\prime}\right|$.

Let $\Theta(x)=0$ if $x<0$ and $\Theta(x)=1$ if $x \geq 0$, and write

$$
\begin{aligned}
F_{A}^{*}(t) & =\sum_{s \in I^{*}(A)} \Theta(t-s), \\
G_{A}^{*}(t) & =\sum_{s \in I^{*}(A)} \Theta(t-|A|+s) .
\end{aligned}
$$

Then

$$
E_{\xi}^{*}\left(A, A^{\prime}, z\right)=\int_{1}^{x_{1}}\left(\int_{0}^{x_{2}} \xi(s+t+z) d F_{A^{\prime}}^{*}(s)\right) d G_{A}^{*}(t) .
$$

From the density property, Definition 2.2(i), we see that

$$
\begin{array}{ll}
\delta_{1}(t-2) \leq G_{A}^{*}(t) \leq t, & t \in\left[1, x_{1}\right] \\
\delta_{1}(s-1) \leq F_{A^{\prime}}^{*}(s) \leq s+1, & s \in\left[0, x_{2}\right] .
\end{array}
$$

Using (4.22) and integrating by parts we find

$$
\begin{aligned}
& E_{\xi}^{*}\left(A, A^{\prime}, y_{1}\right)-E_{\xi}^{*}\left(A, A^{\prime}, 0\right) \\
& \quad \geq A \delta_{1}^{2}\left[\int_{1}^{x_{1}} \int_{0}^{x_{2}}\left(\frac{1}{(s+t)^{2}}-\frac{1}{\left(s+t+y_{1}\right)^{2}}\right) d t d s-3\right] .
\end{aligned}
$$

Similarly,

$$
\begin{aligned}
& E_{\xi}^{*}\left(A^{\prime}, A^{\prime \prime}, y_{2}\right)-E_{\xi}^{*}\left(A^{\prime}, A^{\prime \prime}, y_{1}+y_{2}\right) \\
& \quad \geq-A\left[\int_{1}^{x_{2}} \int_{0}^{\infty}\left(\frac{1}{\left(s+t+y_{2}\right)^{2}}-\frac{1}{\left(s+t+y_{1}+y_{2}\right)^{2}}\right) d t d s-3\right] .
\end{aligned}
$$


Computing the integral in (4.23) and using (4.18) and (4.19) yields

$$
E_{\xi}^{*}\left(A, A^{\prime}, y_{1}\right)-E_{\xi}^{*}\left(A, A^{\prime}, 0\right) \geq A \delta_{1}^{2}\left[\log \frac{(2 \tau+\sigma)(1+\tau)(1+\sigma)}{2(1+\tau+\sigma)(\tau+\sigma)}-3\right]
$$

where $\tau=u_{j-1}^{1 / 5}$ and $\sigma=\delta_{1} u_{j}$. Similarly, from (4.24)

$$
\begin{aligned}
& E_{\xi}^{*}\left(A^{\prime}, A^{\prime \prime}, y_{2}\right)-E_{\xi}^{*}\left(A^{\prime}, A^{\prime \prime}, y_{1}+y_{2}\right) \\
& \quad \geq-A \log \frac{\left(x_{2}-\sigma\right)\left(1+y_{1}+\sigma\right)}{\left(x_{2}+y_{1}+\sigma\right)(1+\sigma)}-3 A .
\end{aligned}
$$

Now, $\delta_{1} \geq 1 / 3$ and $\min \left(x_{2}, y_{1}\right)<u_{j+1}$, so (4.25) and (4.26) give

$$
H_{\xi, 1}^{*(k)}-H_{\xi, 1}^{*(k+1)} \geq A(j / 90-13)
$$

Suppose now that $\eta_{k}=0$. The estimate (4.26) holds also in this case, and since we always have $E_{\xi}^{*}\left(A, A^{\prime}, y_{1}\right)-E_{\xi}^{*}\left(A, A^{\prime}, 0\right) \geq 0$, (4.17) gives

$$
H_{\xi, 1}^{*(k)}-H_{\xi, 1}^{*(k+1)} \geq-6 A
$$

Summing (4.27) and (4.28) over all $k$ and using (4.16) we obtain

$$
\Delta H_{\xi, 1} \geq \sum_{j=1}^{p} \sum_{k=r_{\jmath}-1}^{r_{\jmath}-1} A\left[\eta_{k}(j / 90-13)-6\left(1-\eta_{k}\right)\right]
$$

In order to obtain the estimate we want, we have to prove that we get a sufficient energy gain in the case $\eta_{k}=0$.

Consider the $k^{\text {th }}$ rearrangement, $r_{j-1} \leq k<r_{j}$, and assume that $\eta_{k}=0$. In the $k^{\text {th }}$ rearrangement two $o$-blocks $A=A_{p_{k}} \ldots A_{q_{k}-1}$ and $A^{\prime}=A_{q_{k}} \ldots A_{l_{k}-1}$ are joined. Suppose that $w(A)>2|A|^{5}$; the case $w\left(A^{\prime}\right)>2\left|A^{\prime}\right|^{5}$ is analogous. Let $N(A)$ denote the total number of particles in $A$. From the definition of $w(A)$ it follows that $N(A) \geq w(A)-|A|$. There must be a block $A_{i_{k}}$ in $A$ such that $N_{k}=N\left(A_{\imath_{k}}\right) \geq N(A) /|A|$. The previous inequalities give

$$
N_{k} \geq \frac{w(A)}{2|A|} .
$$

Thus, to each $k$ with $\eta_{k}=0$, we can assign a pair $\left(A_{i_{k}}, A_{q_{k}}\right)$ of o-blocks. Clearly different $k$ 's give different pairs of $o$-blocks. Let $z_{\imath}$ and $\zeta_{i}$ be the distance between the $i^{\text {th }}$ particle in $A_{i_{k}}$ and the first particle in $A_{q_{k}}$ in $\Gamma^{(0)}(\underline{n})$ and $\Gamma^{(s-1)}(\underline{n})$ respectively, $i=1, \ldots, N_{k}$. Then

$$
\begin{aligned}
& z_{\imath} \geq 1+\zeta_{\imath} \\
& \zeta_{\imath} \leq|A|
\end{aligned}
$$

Since $\xi(r)$ is increasing, we get

$$
\Delta H_{\xi, 1} \geq A \sum_{j=1}^{p} \sum_{k=r_{j}-1}^{r_{\jmath}-1} \sum_{i=1}^{N_{k}}\left(-\frac{1}{z_{\imath}^{2}}+\frac{1}{\zeta_{i}^{2}}\right)\left(1-\eta_{k}\right) .
$$


Now, by (4.30)-(4.32),

$$
\begin{aligned}
\sum_{i=1}^{N_{k}}\left(-\frac{1}{z_{i}^{2}}+\frac{1}{\zeta_{i}^{2}}\right) & \geq \sum_{i=1}^{N_{k}} \frac{1}{2 \zeta_{1}^{3}} \geq \frac{N_{k}}{2|A|^{3}} \\
& \geq \frac{2^{4 / 5}}{4} w(A)^{1 / 5} \geq \frac{2^{4 / 5}}{4} u_{j}^{1 / 5}
\end{aligned}
$$

and $u_{j} \geq(\log 2) j / 5$, so

$$
\Delta H_{\xi, 1} \geq A \sum_{j=1}^{p} \sum_{k=r_{\jmath}-1}^{r_{\jmath}-1} j\left(1-\eta_{k}\right) / 90 .
$$

Adding (4.29) and (4.33) we get (4.11) with $\kappa_{2}=7 A$ and $\kappa_{3}=A / 180$.

Proof of (4.12). Since the distance between any two e-blocks $B_{j}$ and $B_{k}$ in $\Gamma^{(0)}$ is greater than the distance between $B_{j}$ and $B_{k}$ in $\Gamma^{(s-1)}$, and since $\phi(r)$ is increasing for $r \geq 1$,

$$
\begin{aligned}
\Delta H_{\phi, 3} \geq & \sum_{k=1}^{s} \sum_{j=1}^{k-1}\left[E_{\phi}\left(B_{j}, A_{k}, a_{j+1, k-1}+b_{j+1, k-1}\right)\right. \\
& \left.-E_{\phi}\left(A_{k}, B_{\jmath}, a_{k+1, s}+b_{1, j-1}\right)\right] \\
& +\sum_{k=1}^{s} \sum_{j=k}^{s}\left[E_{\phi}\left(A_{k}, B_{j}, a_{k+1, j}+b_{k+1, j-1}\right)\right. \\
& \left.-E_{\phi}\left(A_{k}, B_{\jmath}, a_{k+1, s}+b_{1, \jmath-1}\right)\right],
\end{aligned}
$$

where we have used the notation

$$
\begin{gathered}
a_{j, k}=\left|A_{j}\right|+\ldots+\left|A_{k}\right|, \\
b_{j, k}=\left|B_{j}\right|+\ldots+\left|B_{k}\right|
\end{gathered}
$$

for $j \leq l$ and $a_{\jmath, k}=b_{\jmath, k}=0$ for $j>k$. The right-hand side of (4.34) represents the change in the interaction energy between $o$ - and $e$-blocks when going from a configuration to the corresponding condensed configuration.

Using the fact that $E_{\phi}(\cdot, \cdot, \cdot) \leq 0$ and rewriting the second double sum in (4.34) we obtain

$$
\begin{aligned}
\Delta H_{\phi, 3} \geq & \sum_{k=1}^{s} \sum_{j=1}^{k-1} E_{\phi}\left(B_{j}, A_{k}, a_{j+1, k-1}+b_{j+1, k-1}\right) \\
& +\sum_{k=1}^{s} \sum_{j=k}^{s}\left[E_{\phi}\left(A_{k}, B_{j}, a_{k+1, j}+b_{k, j-1}\right)\right. \\
& \left.-E_{\phi}\left(A_{k}, B_{j}, a_{k+1, s}+b_{1, j-1}\right)\right] \\
& +\sum_{k=1}^{s} E_{\phi}\left(A_{k}, B^{(k)}, a_{k+1, s}\right) .
\end{aligned}
$$

where we have written $B^{(k)}=B_{k} \ldots B_{s}$. 
The estimate we want follows from the following three estimates of the terms is the sums in (4.35). If $j<k$, the

$$
\begin{aligned}
E_{\phi}( & \left.B_{j}, A_{k}, a_{j+1, k-1}+b_{j+1, k-1}\right) \\
\geq & -\kappa \sum_{l=1}^{j}\left(E_{\phi}\left(A_{l}, A_{k}, a_{l+1, k-1}+b_{\jmath, k-1}\right)\right. \\
& \left.-E_{\phi}\left(A_{l}, A_{k}, a_{l+1, k-1}+b_{\jmath+1, k-1}\right)\right)
\end{aligned}
$$

where $\kappa=\delta_{2}\left(1-\delta_{2}\right)^{-1} \delta_{1}^{-1}$. If $j \geq k$, then

$$
\begin{aligned}
E_{\phi}( & \left.A_{k}, B_{j}, a_{k+1, j}+b_{k, j-1}\right) \\
& -E_{\phi}\left(A_{k}, B_{j}, a_{k+1, s}+b_{1, j-1}\right) \\
\geq & -\kappa \sum_{l=j+1}^{j}\left(E_{\phi}\left(A_{k}, A_{l}, a_{k+1, l-1}+b_{k, j}\right)\right. \\
& \left.-E_{\phi}\left(A_{k}, A_{l}, a_{k+1, l-1}+b_{k, j-1}\right)\right) .
\end{aligned}
$$

The third estimate is the most difficult one: there is a $Q_{N}^{*} \subseteq Q_{N, \varrho}$ satisfying Lemma 4.1(i) such that if $\underline{m}(\underline{x}) \in Q_{N, \varrho} \backslash Q_{N}^{*}, \varrho<1 / 4$ and $\lambda \geq 7$, then

$$
\sum_{k=1}^{s} E_{\phi}\left(A_{k}, B^{(k)}, a_{k+1, s}\right) \geq-\lambda^{-1} \kappa_{4} \log N,
$$

where $\kappa_{4}$ only depends on the parameters in $\phi$.

The idea behind the above estimates is the following rearrangement procedure. Consider the $o$-block $A_{k}$. Let successively the $e$-blocks $B_{s-1}, B_{s-2}, \ldots, B_{k}$ change place with the $o$-block to the right. Then the interaction between the $e$-blocks and $A_{k}$ goes up, but the interaction energy between $A_{k}$ and the $o$-blocks to the right goes down. We compare these energy changes. Then we let successively the $e$-blocks $B_{1}, \ldots, B_{k-1}$ change place with the $o$-block to the left, and argue similarly with the energy changes. Finally we insert the $e$-blocks $B_{1}, \ldots, B_{k-1}$ between $A_{1} \ldots A_{s}$ and $B_{k} \ldots B_{s}$. This leads to an energy change estimated by (4.38). The proofs of (4.36) and (4.37) are not difficult, but (4.38) is far from immediate. To prove it we need some control over how many particles there are in a box in typical configurations. For example, if there are very many particles, say $(\log N)^{2}$, in the last box in $A_{s}$, then the estimate need not be true. We will prove in Sect. 5 that configurations for which (4.38) fails have a vanishing probability in the limit $N \rightarrow \infty$.

Assume now that (4.36)-(4.38) are valid. Use (4.36) and (4.37) in the first and second sums in (4.35) respectively, rearrange these sums, and use (4.38) to estimate the last sum. This gives (4.11) if $\lambda \geq 7$ (which implies $\kappa \leq 1 / 2$ ).

We will now prove (4.37). The proof of (4.36) is analogous. Inequality (4.38) will be proved in Sect. 5 .

Let $A=A_{j+1} \ldots A_{r}, x=|A|, z=a_{k+1, j}+b_{k, j-1}$ and $y=\left|B_{\jmath}\right|$. Then (4.37) can be written

$$
\begin{aligned}
& E_{\phi}\left(A_{k}, B_{j}, z\right)-E_{\phi}\left(A_{k}, B_{j}, z+x\right) \\
& \quad \geq-\kappa\left[E_{\phi}\left(A_{k}, A, z+y\right)-E_{\phi}\left(A_{k}, A, z\right)\right] .
\end{aligned}
$$


We can write

$$
E_{\phi}\left(C, C^{\prime}, z\right)=\int_{0}^{x}\left(\int_{0}^{x^{\prime}} \phi(t+s+z) d F_{C^{\prime}}(t)\right) d G_{C}(s),
$$

where $F_{C^{\prime}}$ and $G_{C}$ are defined as in (4.28) $I(\cdot)$ given by (4.4) instead of $I^{*}(\cdot)$. Hence we see that (4.39) follows if we can prove that for each $t \geq 0$,

$$
\begin{aligned}
& \int_{0}^{y}(\phi(t+s+z+x)-\phi(t+s+z)) d F_{B_{j}}(s) \\
& \quad \leq \kappa \int_{0}^{x}(\phi(t+s+z+y)-\phi(t+s+z)) d F_{A}(s) .
\end{aligned}
$$

From the density property we now that $F_{A}(s) \geq \delta_{1}(s-1)$ and $F_{B_{j}}(s) \leq \delta_{2} s$, and by definition $F_{B_{j}}(s) \leq 0$ if $s<1 / \delta_{2}$. A consequence of this is that $F_{B_{j}}(s) \leq$ $\delta_{2}\left(1-\delta_{2}\right)^{-1}(s-1)$, if $s \geq 1$. An integration by parts shows that the left-hand side of (4.40) can be written

$$
\begin{aligned}
& -\int_{0}^{y}\left(\phi^{\prime}(t+s+z+x)-\phi^{\prime}(t+s+z)\right) F_{B_{\jmath}}(s) d s \\
& +(\phi(t+y+z+x)-\phi(t+y+z)) F_{B_{j}}(y) \\
& \leq-\delta_{1} \kappa \int_{1}^{y}\left(\phi^{\prime}(t+s+z+x)-\phi^{\prime}(t+s+z)\right)(s-1) d s \\
& +\delta_{1} \kappa[(\phi(t+y+z+x)-\phi(t+y+z))(y-1) \\
& -(\phi(t+z+x)-\phi(t+z))(0-1)] .
\end{aligned}
$$

Here we use the fact that $\phi(r)$ is increasing and $\phi^{\prime}(r)$ is decreasing when $r \geq 1$. The integration by parts formula shows that this equals

$$
\begin{aligned}
& \delta_{1} \kappa \int_{0}^{y}(\phi(t+s+z+x)-\phi(t+s+z)) d s \\
& \quad=\delta_{1} \kappa \int_{0}^{x}(\phi(t+s+z+y)-\phi(t+s+z)) d s .
\end{aligned}
$$

Repeating the argument above, now using the inequality $F_{A}(s) \geq \delta_{1}(s-1)$, we find that the right-hand side of (4.41) is

$$
\leq \kappa \int_{0}^{x}(\phi(t+s+z+y)-\phi(t+s+z)) d F_{A}(s),
$$

and we have proved (4.40). 
Remark 4.2. To prove the existence of a phase separation in an ordinary lattice gas as given in Remark 1.8, one can use methods similar to the ones used above. Assume that $\varrho<1 / r_{0}$ and define $\gamma^{(0)}(\underline{n})$ as follows: $k \in \gamma^{(0)}(\underline{n})$ if $n_{k-r_{0}}=1, n_{k-r_{0}+1}=$ $\ldots=n_{k-1}=n_{k}=0$ or if $n_{k-r_{0}}=\ldots=n_{k-1}=0$ and $n_{k}=1$, i.e. an $o$-block in $\left(\underline{n}, \gamma^{(0)}(\underline{n})\right)$ always ends with $r_{0}-1$ zeros. With this definition $J(r), r<r_{0}$, does not enter into the energy considerations in the rearrangement procedure.

\section{Proof of (4.38)}

In this section our goal is to show that there is a $Q_{N}^{*} \subseteq Q_{N, \varrho}$ such that

$$
P_{N, \varrho, \phi}^{c}\left\{\underline{x} ; \underline{n}(\underline{x}) \in Q_{N}^{*}\right\} 2 \varrho^{-1} N^{2-c_{1}^{*} \beta},
$$

if $\lambda \geq 7$ and $\varrho<1 / 4$, where $c_{1}^{*}$ just depends on the parameters in $\phi$, and if $\underline{x} \in \Omega(\underline{n})$, $\underline{n} \in Q_{N, \varrho} \backslash Q_{N}^{*}$, then (4.38) holds.

What we want to show is that configurations where there is a large number of particles in a single box $[j-1, j$ ), or in a sequence of boxes, has small probability for large $N$. The proof is by an energy-entropy argument.

Let $\nu_{0}$ be a given integer $>2$ and put

$$
Q^{(j)}=\left\{\underline{n} \in Q_{N, \varrho} ; n_{j}>\nu_{0}\right\}, \quad j=1, \ldots, L .
$$

That $\underline{n} \in Q^{(j)}$ means that there is an excess of particles in box $\mathrm{nr} j,[j-1, j)$. The next lemma given us a way of moving a particle from box $j$, which contains an excess of particles, to an empty box. This lemma is the combinatorial part of the argument.

Lemma 5.1. There is an injective map $T_{j}: Q^{(j)} \rightarrow Q_{N, \varrho}$ such that if $\underline{m}=T_{j} \underline{n}$, then $m_{j}=n_{j}-1$ and there is an $l=l(\underline{n})$ such that $n_{l-1}=n_{l}=n_{l+1}=0, m_{l}=1$ and $m_{\imath}=n_{i}$ for $i$ not equal to $j$ or $l$.

Proof. The proof will be given later in this section.

The next step is to use $T_{j}$ to define a map $F: \Omega(\underline{n}) \rightarrow \Omega\left(T_{j} \underline{n}\right)$, and use $F$ in an energy entropy argument to show that the probability of configurations where the number of excess particles in some box is $\geq C(\log N)^{1 / 4+1 / 2 \gamma}$ goes to zero in the limit $N \rightarrow \infty$.

Lemma 5.2. There is a $\nu_{0}>2$ such that if $Q^{(j)}$ is defined by (5.2) the following holds. For each $\underline{n} \in Q^{(j)}, 1 \leq j \leq L$, there is an injective map $F: \Omega(\underline{n}) \rightarrow \Omega\left(T_{j} \underline{n}\right)$ and a partition

$$
\Omega(\underline{n})=\bigcup_{\eta \in I} \Omega(\underline{n}, \eta), \quad(\text { disjoint union })
$$

where $I$ is a finite index set, so that

(i) $F: \Omega(\underline{n}, \eta) \rightarrow F(\Omega(\underline{n}, \eta))$ is a diffeomorphism with Jacobian $=1$, and

(ii) if $\underline{x} \in \Omega(\underline{n})$ and $n_{\imath} \leq n_{\text {J }}$ for $i=1, \ldots, L$, then

$$
H_{\phi}(\underline{x})-H_{\phi}(F(\underline{x})) \geq \frac{B}{8} n_{\jmath}^{\gamma}
$$

where $B$ is the constant in (1.1).

Proof. The proof will be given below. 
We will now prove

Lemma 5.3. Let

$$
\Omega^{\prime}=\left\{\underline{x} \in \Omega: \max _{1 \leq j \leq L} n_{j}(\underline{x}) \leq[\log N]^{1 / 4+1 / 2 \gamma}\right\}
$$

where $\Omega=\Omega_{N, \varrho}$. Then

$$
P_{N, \varrho, \phi}^{c}\left(\Omega \backslash \Omega^{\prime}\right) \leq \exp \left(-\frac{\beta B}{16}[\log N]^{\gamma / 4+1 / 2}\right) .
$$

Proof. Define for $m \geq[\log N]^{1 / 4+1 / 2 \gamma} \doteq m_{0}, 1 \leq j \leq L$,

$$
Q_{m}^{(j)}=\left\{\underline{n} \in Q^{(\jmath)} ; n_{j}=m \text { and } \max _{1 \leq k \leq L} n_{k}=m\right\}
$$

Then

$$
\Omega \backslash \Omega^{\prime}=\bigcup_{j=1}^{L} \bigcup_{m>m_{0}} \bigcup_{\underline{n} \in Q_{m}^{(j)}} \Omega(\underline{n}) .
$$

Let $N$ be sufficiently large, $\underline{n} \in Q_{m}^{(\jmath)}$ and $F$ be the mapping given by Lemma 5.2. Then, by Lemma 5.2(i) and (ii), for each $\mu \in I$,

$$
\begin{aligned}
Z(F(\Omega(\underline{n}, \mu)) & =\int_{F(\Omega(\underline{n}, \mu))} \exp \left(-\beta H_{\phi}(\underline{y})\right) d^{N} y \\
& =\int_{\Omega(\underline{n}, \mu)} \exp \left(-\beta H_{\phi}(F(\underline{x}))\right) d^{N} x \\
& =\int_{\Omega(\underline{n}, \mu)} \exp \left[\beta\left(H_{\phi}(\underline{x})-H_{\phi}(F(\underline{x}))\right)-\beta H_{\phi}(\underline{x})\right] d^{N} x \\
& \geq \exp \left(\beta B m^{\gamma} / 8\right) Z(\Omega(\underline{n}, \mu)) .
\end{aligned}
$$

Summation over $\mu \in I$ gives

$$
Z(\Omega(\underline{n})) \leq \exp \left(-\beta B m^{\gamma} / 8\right) Z(F(\Omega(\underline{n}))) .
$$

Note that $F(\Omega(\underline{n}, \mu)) \cap F(\Omega(\underline{n}, \nu))=\emptyset$ if $\mu \neq \nu$, since $F: \Omega(\underline{n}) \rightarrow F(\Omega(\underline{n}))$ is injective. We now sum (5.5) over all $\underline{n} \in Q_{m}^{(j)}$ to get

$$
Z\left(\bigcup_{\underline{n} \in Q_{m}^{(j)}} \Omega(\underline{n})\right) \leq \exp \left(-\beta B m^{\gamma} / 8\right) Z(\Omega(\underline{n}))
$$

where we have used $F(\Omega(\underline{n})) \subseteq \Omega\left(T_{j} \underline{n}\right)$ and $\Omega\left(T_{j} \underline{n}\right) \cap \Omega\left(T_{j} \underline{m}\right)=\emptyset$ whenever $\underline{m} \neq \underline{n}$ by Lemma 5.1 . Now by (5.4),

$$
Z\left(\Omega \backslash \Omega^{\prime}\right) \leq L \sum_{m=m_{0}+1}^{\infty} \exp \left(-\beta B m^{\gamma} / 8\right) Z(\Omega(\underline{n})) .
$$

Estimating this sum by an integral, we get

$$
P_{N, \varrho, \phi}^{c}\left(\Omega \backslash \Omega^{\prime}\right) \leq \frac{8 L}{\gamma \beta B m_{0}^{\gamma-1}} \exp \left(-\beta B_{0}^{\gamma} / 8\right) \leq \exp \left(-\frac{\beta B}{16}[\log N]^{\gamma / 4+1 / 2}\right) .
$$


The next lemma does not concern the total number of excess particles in a single box, but the total number of excess particles in certain groups of boxes.

Let $\underline{n} \in Q_{N, \varrho}$ be given. We let $\mathscr{C}(\mathscr{B})$ be the set of indices $k \in Z$, such that $n_{k}$ lies in an $o$-block (e-block) in $\Gamma_{1}(\underline{n})$, see (5.12). Put $\mathscr{J}=\mathscr{J}(\underline{n})=\left\{k ; n_{k} \geq \nu_{0}\right\} \cap \mathscr{b}$.

$\mathscr{J}=\left\{k_{1}, \ldots, k_{M}\right\}$, where $k_{1}<\ldots<k_{M}$. Write $M=[\log N] q+r$ with $0 \leq r<[\log N]$, and put $\mathscr{J}^{(0)}=\left\{k_{1}, \ldots, k_{r}\right\}, \mathscr{J}^{(j)}=\left\{k_{a(\jmath-1)+r+1}, \ldots, k_{a \jmath+r}\right\}$, $j=1, \ldots, q$. Set

$$
m_{\jmath}=m_{j}(\underline{n})=\sum_{k \in \mathscr{P}^{(j)}}\left(n_{k}-\nu_{k}\right), \quad j=0, \ldots, q .
$$

Lemma 5.4. Let

$$
\Omega^{\prime \prime}=\left\{\underline{x} \in \Omega: \max _{0 \leq j \leq q(\underline{x})} m_{j}(\underline{n}(\underline{x})) \leq[\log N]\right\} .
$$

Then for all sufficiently large $N$,

$$
P_{N, \varrho, \phi}^{c}\left(\Omega \backslash \Omega^{\prime \prime}\right) \leq 2 \varrho^{-1} N^{2-c_{1}^{*} \beta},
$$

where $c_{1}^{*}$ only depends on the parameters in $\phi$.

Proof. We will prove that

$$
P_{N, \varrho, \phi}^{c}\left(\left(\Omega \backslash \Omega^{\prime \prime}\right) \cap \Omega^{\prime}\right) \leq(\varrho-\varrho / e)^{-1} N^{2-B \nu_{0}^{\gamma} \beta / 24},
$$

which, if we take $c_{1}^{*}=B / 3$, together with (5.3) implies (5.6), since $\nu_{0} \geq 3$ and $\gamma>2$. Given $j, 0 \leq j \leq q$ and $m>[\log N]$, let

$$
L_{j, m}=\left\{\underline{n} \in Q_{N, \varrho} ; \max _{1 \leq \imath \leq L} n_{i} \leq m_{0}, m_{j}(\underline{n})=m \text { and } \max _{0 \leq k \leq q} m_{k}(\underline{n})=m_{j}(\underline{n})\right\} .
$$

Then

$$
\left(\Omega \backslash \Omega^{\prime \prime}\right) \cap \Omega^{\prime}=\bigcup_{j=0}^{q} \bigcup_{m>[\log N]} \bigcup_{n \in L_{j, m}} \Omega(\underline{n}) .
$$

Consider $\underline{n} \in L_{j, m}$. The idea is now to move the excess particles in the boxes with indices in $\mathscr{J}^{(j)}$ successively to empty boxes using the maps defined in Lemma 5.1 and 5.2 .

Let $\underline{n}^{(1)}=\underline{n}$. Suppose that $\underline{n}^{(i)}$ has been defined, and that

$$
\sum_{k \in \mathscr{Z}^{(j)}}\left(n_{k}^{(i)}-\nu_{0}\right)>0
$$

which means that $\underline{n}^{(i)}$ still contains excess particles in boxes with index in $\mathscr{J}^{(j)}$. Let $k_{\tau}$ be the smallest $k$ in $\mathscr{T}^{(j)}$ such that

$$
n_{k_{\tau}}^{(i)}=\max _{k \in \mathscr{J}^{(j)}} n_{k}^{(2)}
$$

Then $\underline{n}^{(\imath)} \in Q^{\left(k_{\tau}\right)}$, and we put

$$
\underline{n}^{(i+1)}=T_{k_{\tau}} \underline{n}^{(\imath)},
$$

with $T_{k_{\tau}}$ given by Lemma 5.1. We also get a map $F_{\imath}: \Omega\left(\underline{n}^{(i)}\right) \rightarrow \Omega\left(\underline{n}^{(i+1)}\right)$ from Lemma 5.2. Furthermore, we define the map $T: L_{j, m} \rightarrow Q_{N, \varrho}$ by $T(\underline{n})=\underline{n}^{(m+1)}$. 
Then there are no excess particles in the boxes with index in $\mathscr{J}^{(j)}$ in $T(\underline{n})$. Note that the map $T: L_{j, m} \rightarrow Q_{N, \varrho}$ is not necessarily injective.

We can now formulate the essential energy and entropy estimates:

Claim 5.5. Let $\underline{x} \in \Omega\left(\underline{n}^{(i)}\right), 1 \leq i \leq m$. There is a number $\lambda_{i}$, independent of $\underline{x}$, such that

$$
H_{\phi}(\underline{x})-H_{\phi}\left(F_{i}(\underline{x})\right) \geq \lambda_{i} .
$$

These numbers satisfy

$$
\sum_{i=1}^{m} \lambda_{i} \geq \frac{B \nu_{0}^{\gamma} m}{24}
$$

Furthermore, for each $\underline{n}^{\prime} \in T\left(L_{\jmath, m}\right)$,

$$
\left|\left\{\underline{n} \in L_{j, m} ; \underline{n}^{\prime}=T \underline{n}\right\}\right| \leq \exp m .
$$

The proof of the claim will be given below.

Assuming the validity of the claim we can now complete the proof of the lemma. By the same argument as in the proof on Lemma 5.3 we see that

$$
Z\left(\Omega\left(\underline{n}^{(i+1)}\right)\right) \geq Z\left(F_{i}\left(\Omega\left(\underline{n}^{(i)}\right)\right)\right) \geq e^{\beta \lambda_{\imath}} Z\left(\Omega\left(\underline{n}^{(\imath)}\right)\right) .
$$

Here we have used (5.8). Repeated use of this inequality gives

$$
\begin{aligned}
Z(\Omega(\underline{n})) & \leq \exp \left(-\beta \sum_{\imath=1}^{m} \lambda_{\imath}\right) Z(\Omega(T \underline{n})) \\
& \leq \exp \left(-C_{1} \beta m\right) Z(\Omega(T \underline{n})),
\end{aligned}
$$

where the second inequality follows from (5.9), and $C_{1}=B \nu_{0}^{\gamma} / 24$. Using this we find

$$
\begin{aligned}
\sum_{\underline{n} \in L_{j, m}} Z(\Omega(\underline{n})) & \leq e^{C_{1} \beta m} \sum_{\underline{n} \in L_{j, m}} Z(\Omega(T \underline{n})) \\
& =e^{-C_{1} \beta m} \sum_{\underline{n}^{\prime} \in T\left(L_{j, m}\right)} Z\left(\Omega\left(\underline{n}^{\prime}\right)\right)\left|\left\{\underline{n} \in L_{j, m} ; \underline{n}^{\prime}=T \underline{n}\right\}\right| \\
& \leq e^{-m\left(C_{1} \beta-1\right)} \sum_{\underline{n}^{\prime} \in T\left(L_{j, m}\right)} Z\left(\Omega\left(\underline{n}^{\prime}\right)\right) \leq e^{-m\left(C_{1} \beta-1\right)} Z(\Omega) .
\end{aligned}
$$

If $2 \geq C_{1} \beta$ the estimate (5.6) is trivial, so we can assume that $C_{1} \beta>2$. Then (5.7) and (5.11) give

$$
P_{N, \varrho, \phi}^{c}\left(\left(\Omega \backslash \Omega^{\prime \prime}\right) \cap \Omega^{\prime}\right) \leq \sum_{m=[\log N]+1}^{\infty} e^{-m\left(C_{1} 1 \beta-1\right)} \leq \frac{1 / \varrho}{1-e^{\left.-C_{1} \beta-1\right)}} e^{-\left(C_{1} \beta-2\right)[\log N]} .
$$

This completes the proof of Lemma 5.4.

The results needed to prove (4.38) are now at our disposal, so we proceed with

Proof of (4.38). With the notation introduced above let

$$
Q_{N}^{*}=\left\{\underline{n} \in Q_{N, \varrho} ; \max _{0 \leq j \leq q} m_{j}(\underline{n})>[\log N]\right\} .
$$


Then (5.1) holds by Lemma 5.4. We have to show that the inequality (4.38) holds for all $\underline{x}$ with $\underline{n}(\underline{x}) \in Q_{N, \varrho} \backslash Q_{N}^{*}$.

Let $\underline{n} \in \Theta_{N, \varrho} \backslash Q_{N}^{*}$. $\left(\underline{n}, \Gamma_{1}(\underline{n})\right)$ gives a partition of $\underline{n}=A_{1} B_{1} \ldots A_{s} B_{s}$, where $A_{k}=\left\langle a_{k}, b_{k}\right\rangle, k=1, \ldots, s$ are the $o$-blocks. Put

$$
\mathscr{A}=\bigcup_{k=1}^{s}\left\{j \in \mathbb{Z} ; a_{k} \leq j<b_{k}\right\},
$$

so that $\mathscr{A}=\left\{\ldots, l_{-1}, l_{0}, \ldots, l_{T}\right\}, l_{\imath}<l_{i+1}$ is the set of all indices for boxes in $o$-blocks. Write $\mu_{i}=n_{l_{T-i}}, i \geq 0$, to simplify the notation. Let $\mathscr{J}^{\prime}=\mathscr{b} \backslash \mathscr{J}$. Then $\mu_{i}<\nu_{0}$ if $i \in \mathscr{J}^{\prime}$.

Observe that the interaction energy between two boxes at distance $z$ from each other containing $\nu$ and $\mu$ particles respectively is $\geq-A \mu \nu / z^{2}$. Recall that $B^{(k)}=B_{k} \ldots B_{s}$ and write $B^{(k)}=\left(\xi_{1}, \xi_{2}, \ldots\right)$, i.e. $\xi_{i}$ is the number of particles in the $i^{\text {th }}$ box in $B^{(k)}$. Then $\xi_{1}=0$ and $\xi_{i}=0$ if $i>L$. It follows from the density property that

$$
\sum_{k=1}^{m} \xi_{k} \leq \delta_{2} m, \quad 1 \leq m \leq L
$$

If $x_{k}=\left|A_{k}\right|$, we have

$$
\begin{aligned}
E_{\phi}\left(A_{k}, B^{(k)}, a_{k+1, s}\right) & \geq-A \sum_{i=1}^{x_{k}} \sum_{t=2}^{L} \frac{n_{b_{k-i}} \xi_{t}}{\left(a_{k+1, s}-2+i+t\right)^{2}} \\
& \geq-A \delta_{2} \sum_{i=1}^{x_{k}} \sum_{t=1}^{L} \frac{n_{b_{k}-\imath}}{\left(a_{k+1, s}-1+i+t\right)^{2}},
\end{aligned}
$$

where we have used (5.13) to get the second inequality. Now, since $a_{k+1, s}+x_{k}=a_{k, s}$, $a_{s+1, s}=0$ and $n_{l_{T}}=0$, we get

$$
\begin{aligned}
\sum_{k=1}^{s} E_{\phi}\left(A_{k}, B^{(k)}, a_{k+1, s}\right) & \geq-A \delta_{2} \sum_{t=1}^{L} \sum_{i=1}^{\infty} \frac{\mu_{i}}{(i+t)^{2}} \\
& =-A \delta_{2} \sum_{t=1}^{L}\left[\sum_{i \in \mathscr{F}^{\prime}} \frac{\mu_{i}}{(i+t)^{2}}+\sum_{i \in \mathscr{F}} \frac{\nu_{0}}{(i+t)^{2}}+\sum_{i \in \mathscr{F}} \frac{\mu_{i}-\nu_{0}}{(i+t)^{2}}\right] \\
& \geq-A \delta_{2} \sum_{t=1}^{L}\left[\sum_{i=1}^{\infty} \frac{\nu_{0}}{(i+t)^{2}}+\sum_{j=0}^{q} \sum_{i \in \mathscr{J}^{(j)}} \frac{\mu_{i}-\nu_{0}}{(i+t)^{2}}\right] \\
& \geq-A \delta_{2}\left[\nu_{0} \log L+\sum_{t=1}^{L} \sum_{j=0}^{q} \frac{m_{j}}{([\log N] j+t)^{2}}\right]
\end{aligned}
$$

where we have used $\mu_{i} \leq \nu_{0}$ when $i \in \mathscr{J}^{\prime}, m_{\imath}=\sum_{i \in \mathscr{J}^{(j)}}\left(\mu_{i}-\nu_{0}\right)$, and $\left|\mathscr{J}^{(j)}\right|=$ $[\log N], j=1, \ldots, q$ and $\left|\mathscr{J}^{(0)}\right| \leq[\log N]$. Since $\underline{n} \in Q_{N}^{*}, m_{i} \leq[\log N]$ and hence 
the last expression in (5.15) is bounded from below by

$$
\begin{aligned}
& -A \delta_{2}\left[\nu_{0} \log L+[\log N] \sum_{t=1}^{\infty} \sum_{j=0}^{q} \frac{1}{([\log N] j+t)^{2}}\right] \\
\geq & -A \delta_{2}\left[\nu_{0} \log L+[\log N] \sum_{j=1}^{q} \frac{1}{([\log N] j)^{2}}\right] \\
\geq & -A \delta_{2}\left[\nu_{0} \log L+\log q+C\right] .
\end{aligned}
$$

Now $L=N / \varrho$ and $q \leq N /[\log N]$, so when $N$ is sufficiently large we have

$$
\sum_{k=1}^{s} E_{\phi}\left(A_{k}, B^{(k)}, a_{k+1, s}\right) \geq-A \delta_{2}\left(\nu_{0}+1\right) \log N,
$$

which proves (4.38) with $\kappa_{4}=A\left(\nu_{0}+1\right)$.

Proof of Lemma 5.1. Let $s, t \in[0, L]$ be two integers and let $Q_{s, t}^{(j)}$ be the set of all $\underline{n}$ in $Q^{(j)}$ such that the number of boxes with exactly one particle is $=s$ and the number of empty boxes is $=t$. Let $\tau$ be the number of $n_{k}$ 's such that $n_{k-1}=n_{k}=n_{k+1}=0$; $\tau \geq t$. The condition $\varrho<1 / 4$ ensures that $Q_{s, t}^{(j)}=\emptyset$ unless $s+1 \geq \tau$. To simplify the notation we write $X=Q_{s, t}^{(j)}$.

Given $\underline{n} \in X$ we can assign $t$ different $\underline{m} \in Q_{N, \varrho}$ by letting $m_{j}=n_{\jmath}-1, m_{l}=1$ for some $\bar{l}$ such that $n_{l-1}=n_{l}=n_{l+1}=\overline{0}$, and $m_{\imath}=n_{i}$ for $i$ not equal to $l$ or $j$. Let $Y$ be the set of all $\underline{m}$ that can be obtained in this way, starting from any $\underline{n} \in X$. This construction defines a bipartite graph $(X, Y, \mathscr{A})$, where $(\underline{n}, \underline{m}) \in \mathscr{A}$ if $\underline{m}$ can be obtained from $\underline{n}$ in the way described above. Let $\varrho(x)$ denote the valence of the corner $x$ in the graph, and for $A \subseteq X$ let

$$
R(A)=\{\underline{m} \in Y ;(\underline{n}, \underline{m}) \in \mathscr{A} \text { for some } \underline{n} \in A\} .
$$

Given $\underline{m} \in Y$ we can obtain any $\underline{n} \in X$ such that $(\underline{n}, \underline{m}) \in \mathscr{b}$ by moving a particle from one of the $s+1$ boxes with exactly one particle in $\underline{m}$, to the $j^{\text {th }}$ box. Hence $\varrho(\underline{m})=s+1$ and clearly $\varrho(\underline{n})=\tau$ for any $\underline{n} \in X$. Let $A \subseteq X$ be arbitrary. Then

$$
(s+1)|A| \leq \tau|A|=\sum_{\underline{n} \in A} \varrho(\underline{n}) \leq \sum_{\underline{m} \in R(A)} \varrho(\underline{m})=(s+1)|R(A)| .
$$

Hence $|A| \leq|R(A)|$ for all $A \subseteq X$ and according to Hall's lemma ("marriage lemma"), see [GW], there is a matching of $X$ into $Y$, i.e. an injective map $T_{j}: X \rightarrow Y$, such that $\left(\underline{n}, T_{n} \underline{n}\right) \in \mathscr{A}$ for each $\underline{n} \in X$. This gives an injective map $T_{\jmath}: Q_{s, t}^{(j)} \rightarrow Q_{N, \varrho}$. If $\underline{m} \in T_{j}\left(Q_{s, t}^{(j)}\right)$, then $\underline{m}$ has exactly $s+1$ boxes with one particle and $t-1$ empty boxes. Consequently $T_{j}\left(Q_{s, t}^{(j)}\right) \neq T_{j}\left(Q_{s^{\prime}, t^{\prime}}^{(j)}\right)$ if $(s, t) \neq\left(s^{\prime}, t^{\prime}\right)$. Hence $T_{j}: Q^{(\jmath)} \rightarrow Q_{N, \varrho}$ is injective.

Proof of Lemma 5.2. Let $\underline{n} \in Q^{(j)}$ so that $n_{j}<\nu_{0}$; the choice of $\nu_{0}$ will be specified below. Divide box no. $j,[j-1, j)$, into $n_{j}-1$ subboxes, $I_{j}^{(i)}=$ $\left[(j-1)+(i-1) /\left(n_{\jmath}-1\right),(j-1)+i /\left(n_{j}-1\right)\right)$, of length $1 /\left(n_{j}-1\right)$. At least one of these subboxes must contain more than one particle. Let $k=k(\underline{n})$ be given by Lemma 5.1. Let $\Omega(\underline{n}, l, \mu, \nu)$ be the set of all $\underline{x} \in \Omega(\underline{n})$ such that 
$(\bullet)$ each of $I_{j}^{(i)}, 1 \leq i<l$, contains at most one particle, but $I_{j}^{(\imath)}$ contains two or more particles,

$(\bullet) x_{\mu}$ is the first particle in $I_{j}^{(l)}$, and

(•) $x_{\nu}<k(\underline{n})-2, x_{\nu+1} \geq k(\underline{n})+1$.

The index set $I$ is the set of all possible $(l, \mu, \nu)$ for a given $\underline{n}$. We define $F$ on $\Omega(\underline{n}, l, \mu, \nu)$ by $\underline{y}=F(\underline{x})$, where (suppose $\nu>\mu, \nu<\mu$ is analogous)

$$
y_{\imath}= \begin{cases}x_{\imath} & \text { if } 1 \leq i<\mu \text { or } \nu<i \leq N \\ x_{i+1} & \text { if } \mu \leq i<\nu \\ x_{\mu}-(j-1)+k(\underline{n}) & \text { if } i=\nu\end{cases}
$$

It is clear from the definition that (i) is satisfied, and it is also immediate that $F(\Omega(\underline{n}, l, \mu, \nu))$ are disjoint for different values of $(l, \mu, \nu)$, so $F$ is one-to-one on $\Omega(\underline{n})$. From Definition (5.17) of $F$ and the definition of $T_{j}$ in Lemma 5.1 we see that $F$ maps $\Omega(\underline{n})$ to $\Omega\left(T_{j} \underline{n}\right)$.

We now turn to the proof of the energy estimate (ii). Assume that $x_{\sigma}, \ldots, x_{\tau} \in$ $[j-1, j)$ are the positions of the particles in box $j, \tau=\sigma+n_{j}-1$. Define

$$
H_{j}^{(1)}(\underline{x})=\sum_{\sigma \leq i<k \leq \tau} \phi\left(\left|x_{k}-x_{i}\right|\right)
$$

and

$$
H_{j}^{(2)}(\underline{x})=\sum_{i=\sigma}^{\tau} \sum_{k<\sigma \text { or } k>\tau} \phi\left(\left|x_{k}-x_{i}\right|\right)
$$

so that $H_{j}^{(1)}(\underline{x})$ is the interaction energy of the particles in box $j$ with each other, and $H_{j}^{(2)}(\underline{x})$ is the interaction energy between the particles in box $j$ and the particles in all the other boxes. $H_{i}^{(1)}(\underline{x})$ and $H_{i}^{(2)}(\underline{x})$ for $i \neq j$ are defined analogously, and $H_{i}^{(1)}(\underline{x})=0$ if $n_{i} \leq 1$.

The potential $\phi(r)$ in (1.1) satisfies $\phi(r) \leq 0$ if $r \geq 1$. Thus

$$
H_{k(\underline{n})}^{(2)}(F(\underline{x})) \leq 0 \text {. }
$$

We will now prove the estimate

$$
H_{j}^{(1)}(\underline{x})-H_{j}^{(1)}(F(\underline{x})) \geq \frac{B}{4} n_{\jmath}^{\gamma} .
$$

Suppose that $\underline{x} \in \Omega(\underline{n}, l, \mu, \nu)$. Then

$$
H_{j}^{(1)}(\underline{x})-H_{j}^{(1)}(F(\underline{x}))=\sum_{\substack{i=\sigma \\ i \neq \mu, \mu+1}}^{\tau} \phi\left(\left|x_{\jmath}-x_{\mu}\right|\right)+\phi\left(x_{\mu+1}-x_{\mu}\right) .
$$

Recall that $\phi(r)=B / r^{\gamma}-2 A / r^{2}, \gamma>2$. There is a constant $d_{1}>0$ such that $\phi(r) \geq-d_{1}$ for all $r \geq 0$, and there is an $r_{0}$ such that

$$
\phi(r) \geq B / 2 r^{\gamma}, \quad \text { if } 0<r \leq r_{0}
$$


and $\phi(r)$ is decreasing in $\left(0, r_{0}\right)$. Let $\nu_{0,1}=\left[1 / r_{0}\right]+1$. Since $x_{\mu+1}-x_{\mu} \leq 1 /\left(n_{j}-1\right)$, we see that if $\nu_{0} \geq \nu_{0,1}$, then $n_{j}-1 \geq \nu_{0,1}$ and thus $x_{\mu+1}-x_{\mu} \leq r_{0}$. Hence (5.20) and (5.21) give

$$
\begin{aligned}
H_{j}^{(1)}(\underline{x})-H_{j}^{(1)}(F(\underline{x})) & \geq-d_{1} n_{j}+\frac{B}{2}\left(n_{j}-1\right)^{\gamma} \\
& \geq n_{j}^{\gamma}\left[-\frac{d_{1}}{\nu_{0}^{\gamma-1}}+\frac{B}{2}\left(1-\frac{1}{\nu_{0}}\right)\right] .
\end{aligned}
$$

Choose $\nu_{0,2}$ so that if $\nu_{0} \geq \nu_{0,2}$ then the expression in brackets in the last line in (5.22) is $\geq B / 4$. This proves (5.19) if $\nu_{0} \geq \max \left(\nu_{0,1}, \nu_{0,2}\right)$.

The next step is to estimate

$$
H_{\jmath}^{(2)}(\underline{x})-H_{j}^{(2)}(F(\underline{x}))=\sum_{k<\sigma \text { or } k>\tau} \phi\left(\left|x_{k}-x_{\mu}\right|\right) .
$$

The boxes adjacent with box $j$ each contains $\leq n_{j}$ particles by assumption. Hence their contribution to the sum in (5.23) is $\geq-2 d_{1} n_{\jmath}$. The distance from a particle in box $k$ to the partticle at $x_{\mu}$ is $\geq|k-j|-1$. Since the number of particles in any box is $\leq n_{j}$ and $\phi(r) \geq 2 A / r^{2}$ we see that

$$
H_{\jmath}^{(2)}(\underline{x})-H_{j}^{(2)}(F(\underline{x})) \geq-2 d_{1} n_{\jmath}-2 n_{j} \sum_{k=1}^{\infty} \frac{2 A}{k^{2}}=-d_{2} n_{j},
$$

where $d_{2}=2 d_{1}+2 A \pi^{2} / 3$. The estimates (5.18), (5.19) and (5.24) together show that

$$
H_{\phi}(\underline{x})-H_{\phi}(F(\underline{x})) \geq \frac{B}{4} N_{j}^{\gamma}-d_{2} n_{j} \geq \frac{B}{4} n_{j}^{\gamma}\left(1-\frac{4 d_{2}}{B} \nu_{0}^{1-\gamma}\right) .
$$

Choose $\nu_{0,3}$ so that $1-4 d_{2} B^{-1} \nu_{0}^{1-\gamma} \leq 1 / 2$ if $\nu_{0} \geq \nu_{0,3}$. Then the desired inequality follows if $\nu_{0} \geq \max _{1 \leq i \leq 3} \nu_{0, \imath}$, which only depends on the parameters in $\phi$.

Proof of Claim 5.5. We will denote the number of excess particles in the boxes with index in $\mathscr{J}^{(j)}$ by $\nu_{i}, \nu_{i}=\nu_{k_{(j-1)[\log N]+\imath+r}}-\nu_{0}, i=1, \ldots,[\log N]$. Then $\sum_{i=1}^{[\log N]} \nu_{i}=m$. The positions of the particles in box $k$ are $x_{\sigma_{k}}, \ldots, x_{\sigma_{k+1}-1}$, i.e., $k-1 \leq x_{\sigma_{k}}<\ldots<x_{\sigma_{k+1}-1}<k, \sigma_{k+1}=\sigma_{k}$ if there are no particles in box $k$. The interaction energy between the particles in box $k$ and the particles in box $l$ is

$$
H_{k, l}(\underline{x})=\sum_{t=\sigma_{k}}^{\sigma_{k+1}-1} \sum_{\tau=\sigma_{l}, \tau \neq t}^{\sigma_{l+1}-1} \phi\left(\left[x_{\tau}-x_{t} \mid\right) .\right.
$$

By the same argument as in the proof of Lemma 5.2 we can show that

$$
\sum_{l \in \mathscr{J}^{(j)}}\left[H_{k_{\tau}, l}(\underline{x})-H_{k_{\tau}, l}\left(F_{i}(\underline{x})\right)\right] \geq \frac{B}{8}\left(n_{k_{\tau}}^{(i)}\right)^{\gamma}
$$


using the notation introduced in the proof of Lemma 5.4. If $j \in \mathscr{C} \backslash \mathscr{J}$ then $n_{j} \leq \nu_{0}$ by definition. Combining this with the density property for $e$-blocks we see that

$$
\sum_{\mathscr{B} \cup(, \mathscr{\ell} \backslash \mathscr{F})}\left(H_{k_{\tau}, l}(\underline{x})-H_{k_{\tau}, l}\left(F_{\imath}(\underline{x})\right)\right) \geq-4 A \nu_{0}\left(\sum_{j=1}^{\infty} \frac{1}{j^{2}}+d_{1}\right)=-d_{3} \nu_{0},
$$

where $d_{3}=4 A\left(\pi^{2} / 6+d_{1}\right)$.

Consider now

$$
\begin{aligned}
& \sum_{\mathscr{J} \backslash \mathscr{F}^{(j)}}\left[H_{k_{\tau}, l}(\underline{x})-H_{k_{\tau}, l}\left(F_{i}(\underline{x})\right)\right] \\
& \quad=\left(\sum_{t=1}^{[\log N](j-1)+r}+\sum_{t=[\log N] j+r+1}^{M}\right)\left[H_{k_{\tau}, k_{t}}(\underline{x})-H_{k_{\tau}, k_{t}}\left(F_{i}(\underline{x})\right)\right] \\
& \quad \doteq \Sigma_{1}+\Sigma_{2} .
\end{aligned}
$$

If $\left|k_{\tau}-k_{t}\right|=1$ we use the fact that $\max n_{i} \leq m_{0}=[\log N]^{1 / 4+1 / 2 \gamma}$ as above, to get the estimate

$$
\left.H_{k_{\tau}, k_{t}}(\underline{x})-H_{k_{\tau}, k_{t}}\left(F_{\imath} \underline{x}\right)\right) \geq-d_{1} m_{0},
$$

where $d_{1}$ is a lower bound on $\phi(r)$ as above. If $\left|k_{\tau}-k_{t}\right|>1$ we use the fact that the distance between any particle in box $k_{\tau}$ and any particle in box $k_{t}$ is $\geq|\tau-t|$. Combining this with $\max n_{j} \leq m_{0}$ and $\phi(r) \geq-2 A / r^{2}$ we find that in this case

$$
H_{k_{\tau}, k_{t}}(\underline{x})-H_{k_{\tau}, k_{t}}\left(F_{i}(\underline{x})\right) \geq-\frac{2 A m_{0}}{|\tau-t|^{2}} .
$$

Let $d_{4}=\max \left(2 A, d_{1}\right)$. Then (5.28) and (5.29) give

$$
\Sigma_{1} \geq-d_{4} m_{0} \sum_{t=1}^{[\log N](j-1)+r} \frac{1}{(\tau-t)^{2}} \geq-2 d_{4} m_{0} \frac{1}{\tau-[\log N](j-1)-r} .
$$

(Recall that $[\log N](j-1)+r<\tau \leq[\log N] j+r$.) Similarly,

$$
\Sigma_{2} \geq-2 d_{4} m_{0} \frac{1}{[\log N] j+r+1-\tau} .
$$

Combining (5.25)-(5.27), (5.30), (5.31) and (5.18) we obtain

$$
\begin{aligned}
& H_{\phi}(\underline{x})-H_{\phi}\left(F_{\imath}(\underline{x})\right) \\
& \quad \geq \frac{B}{8}\left(n_{k_{\tau}}^{(i)}\right)^{\gamma}-2 d_{4}\left(m_{0}\left(\frac{1}{\tau-[\log N](j-1)-r}+\frac{1}{[\log N] j+r+1-\tau}\right)-d_{3} \nu_{0}\right. \\
& \quad \doteq \lambda_{\imath} .
\end{aligned}
$$

What we do in the construction of $\underline{n}^{(2)}, i=1, \ldots, m$, is that we successively remove the excess particles in the boxes given by $\mathscr{J}^{(j)}$. Hence it follows from (5.32) that

$$
\begin{aligned}
\sum_{i=1}^{m} \lambda_{i} \geq & \frac{B}{8} \sum_{i=1}^{[\log N]} \sum_{j=1}^{\nu_{\imath}}\left(j+\nu_{0}\right)^{\gamma} \\
& +2 d_{4} m_{0} \sum_{i=1}^{[\log N]} \nu_{\imath}\left(\frac{1}{i}+\frac{1}{[\log N]+1-i}\right)-d_{3} \nu_{0} m
\end{aligned}
$$


We will use the following estimate

$$
\begin{aligned}
\sum_{i=1}^{[\log N]} \sum_{j=1}^{\nu_{i}}\left[\left(j+\nu_{0}\right)^{\gamma}-j^{\gamma}\right] & \geq \frac{1}{\gamma+1} \sum_{i=1}^{[\log N]}\left[\left(\nu_{i}+\nu_{0}\right)^{\gamma+1}-\nu_{i}^{\gamma+1}-\nu_{0}^{\gamma+1}\right] \\
& \geq \frac{\gamma}{\gamma+1} \nu_{0}^{\gamma} m \geq \frac{2}{3} \nu_{0}^{\gamma} m
\end{aligned}
$$

Choose $\nu_{0,4}$ so that $\nu_{0} \geq \nu_{0,4}$ implies $B \nu_{0}^{\gamma} / 24-d_{3} \nu_{0} \geq 0$. Them (5.33) and (5.34) imply

$$
\sum_{i=1}^{m} \lambda_{\imath} \geq \frac{B}{24} \nu_{0}^{\gamma} m
$$

if we can show that

$$
\frac{B}{8} \sum_{i=1}^{[\log N]} \sum_{j=1}^{\nu_{\imath}} j^{\gamma}-2 d_{4} m_{0} \sum_{i=1}^{[\log N]} \nu_{i}\left(\frac{1}{i}+\frac{1}{[\log N]+1-i}\right) \geq 0
$$

Using the Cauchy-Schwarz' inequality and the estimate $\sum_{i=1}^{\nu_{2}} j^{\gamma} \geq \nu_{i}^{2}$, we see that the left-hand side of (5.5) is bounded from below by

$$
\frac{B}{8} \sum_{i=1}^{[\log N]} \nu_{i}^{2}-d_{5} m_{0}\left(\sum_{i=1}^{[\log N]} \nu_{i}^{2}\right)^{1 / 2}
$$

where $d_{5}=4 \pi d_{4} / \sqrt{6}$. Now, again by the Cauchy-Schwarz' inequality

$$
m=\sum_{i=1}^{[\log N]} \nu_{\imath} \leq\left(\sum_{i=1}^{[\log N]} \nu_{\imath}^{2}\right)^{1 / 2}[\log N]^{1 / 2}
$$

If we write $x=\left(\sum_{i=1}^{[\log N]} \nu_{i}^{2}\right)^{1 / 2}$ we thus have $x \geq m[\log N]^{-1 / 2}$ and (5.36) equals $g(x)=B x^{2} / 8-d_{5} m_{0} x$. When $N$ is sufficiently large then

$$
\begin{aligned}
g(x) & \geq g\left(m[\log N]^{-1 / 2}\right)=\frac{B}{8} m\left[m[\log N]^{-1}-d_{5} m_{0}[\log N]^{-1 / 2}\right] \\
& \geq \frac{B}{8} m\left(1-8 d_{5}[\log N]^{1 / 2 \gamma-1 / 4}\right) \geq \frac{B}{16} m,
\end{aligned}
$$

since $\gamma>2$ and $m \geq[\log N]$. This proves (5.36).

The condition on $\nu_{0}$ is

$$
\nu_{0} \geq \max _{1 \leq i \leq 4} \nu_{0, \imath},
$$

which only depends on the parameters in $\phi$.

It still remains to prove (5.10). Let $\underline{n} \in L_{j, m}$. There is a sequence $\left\{j_{r}\right\}_{r=1}^{m} \subseteq$ $\mathscr{J}^{(j)}(\underline{n})$, which depends on $\underline{n}$, such that

$$
T \underline{n}=\left(T_{j_{m}} \circ T_{j_{m-1}} \circ \ldots \circ T_{j_{1}}\right)(\underline{n}) .
$$


This means that we first move a particle from box $j_{1}$, then from box $j_{2}$, and so on. Given $\underline{n}^{\prime}=T \underline{n}$ and $\left\{j_{r}\right\}_{r=1}^{m}$ we can find $\underline{n}$ by reversing (5.38). Given $\underline{n}^{\prime}$ we also know $\mathscr{J}^{(j)}(\underline{n})$, since $\mathscr{J}^{(j)}(\underline{n})=\mathscr{J}^{(j)}\left(\underline{n}^{\prime}\right)$. Since $\left|\mathscr{J}^{(j)}(\underline{n})\right|=m$, the number of possible sequences $\left\{j_{r}\right\}_{r=1}^{m}$ is

$$
\left(\begin{array}{c}
m+[\log N]-1 \\
m
\end{array}\right)
$$

and consequently,

$$
\begin{aligned}
\left|\left\{\underline{n} \in L_{j, m} ; \underline{n}^{\prime}=T \underline{n}\right\}\right| & \leq\left(\begin{array}{c}
m+[\log N]-1 \\
m
\end{array}\right) \\
& \leq \exp \left(m \log \frac{m+[\log N]-1}{m}\right) \leq \exp ([\log N]) \leq \exp m
\end{aligned}
$$

Remarks on the Proof of Corollary 1.3. This proof is analogous to the proof of Theorem 1.3 in [Jo1]. The only difference is that when we divide $\Lambda$ into two parts $[0, \xi) \cap \Lambda$ and $[\xi, L] \cap \Lambda$, the interaction energy between the two parts is not $o(N)$ for all configurations. But if we exclude configurations for which the number of particles in a box is $\geq[\log N]^{1 / 4+1 / 2 \gamma}$, which we can do by Lemma 5.3 , it is clear that the interaction energy between the two parts is $o(N)$, and the proof in [Jo1] can be used.

Acknowledgements. Part of this work has been supported by the Swedish Natural Research Council (NFR). I also thank the Department of Mathematics at University of Uppsala for financial support during a part of this project.

\section{References}

[ACCN] Aizenman, M., Chayes, J.T., Chayes, L., Newman, C.M.: Discontinuity of the magnetization in one-dimensional Ising and Potts models. J. Stat. Phys. 50, 1 (1988)

[GW] Graver, E., Watkins M.E.: Combinatorics with emphasis on the theory of graphs. Berlin, Heidelberg, New York: Springer, 1977

[IN] Imbrie, J.Z., Newman, C.M.: An intermediate phase with slow decay of correlations in one-dimensional $1 /|x-y|^{2}$ percolation, Ising and Potts models. Commun. Math. Phys. 118, 303-336 (1988)

[Is] Israel, R.B.: Convexity in the theory of lattice gases. Princeton, NJ: Princeton University Press, 1979

[Jo1] Johansson, K.: Condensation of a one-dimensional lattice gas. Commun. Math. Phys. 141, 41-61 (1991)

[Jo2] Johansson, K.: Separation of phases at low temperatures in a one-dimensional continuous gas. Commun. Math. Phys. 141, 259-278 (1991)

[KY] Klein, D., Yang, W.S.: A characterization of first order phase transitions for superstable interactions in classical statistical mechanics. J. Stat. Phys. 71, 1043-1062 (1993)

[Ru1] Ruelle, D.: Statistical mechanics - rigorous results. New York: Benjamin, 1969

[Ru2] Ruelle, D.: Existence of a phase transition in a continuous classical system. Phys. Rev. Lett. 27, 1040-1041 (1971)

[Si] Simon, B.: Fiften problems in mathematical physics. In: Perspectives in Mathematics, Anniversary of Oberwolfach. Basel: Birkhäuser, 1984 
